\title{
GUANACOS EN EL SEGUNDO COMPONENTE DE TÚNEL I (CANAL BEAGLE). UN ENFOQUE TAFONÓMICO
}

MARTÍN VÁZQUEZ

\section{RESUMEN}

El presente artículo ofrece los resultados del análisis de los restos de guanacos (Lama guanicoe) provenientes del Segundo Componente de Túnel I, emplazado en la margen norte del canal Beagle, particularmente los incluidos en la capa D datada entre 6.400 y 4.500 años AP. Se utiliza un enfoque tafonómico a fin de identificar qué agentes y procesos, y en qué grado, intervinieron en la conformación del conjunto. Con este objetivo en mente se aplica una serie de procedimientos metodológicos y modelos tafonómicos no utilizados previamente en contextos de la región, que a su vez son evaluados en cuanto a su potencial analítico y explicativo en este caso. Se pone especial énfasis en la evaluación de distintas modalidades de cuantificación anatómica y en la aplicación del Modelo de Alto y Bajo Potencial de Supervivencia, las conclusiones han resultado estimulantes para futuras aplicaciones en conjuntos de similares características. En cuanto a las inferencias arqueológicas derivadas de los análisis, se destaca que las cacerías de guanacos se habrían realizado en escala reducida, obteniendo una o pocas presas por evento, las que habrian sido explotadas de forma completa. La representación anatómica diferencial y la frecuencia y variabilidad de huellas antrópicas, entre otras evidencias, sugiere el transporte diferencial de los miembros, el faenamiento intensivo de las presas y la posición terminal de Túnel I en la cadena de explotación de este recurso. Aunque también la dinámica de procesos atricionales, particularmente la acción de carnívoros, habrían tenido una incidencia significativa en la conservación diferencial de elementos.

PALABRAS CLAVE: guanaco, Túnel I, canal Beagle, zooarqueologia, tafonomía.

\section{GUANACOS IN THE SECOND COMPONENT OF TÚNEL I (BEAGLE CHANNEL). A TAPHONOMIC APPROACH}

\footnotetext{
ABSTRACT

This article provides the results of the analysis of guanaco (Lama guanicoe) remains from Second Component of Tunel I site -northern shore of the Beagle Channel- particularly those included in the D layer (6.400-4.500 years AP). A taphonomic approach is used to identify which agents and processes 
took part in the formation of the assemblage, and to what degree. With this purpose in mind, a series of methodological procedures and taphonomic models not previously used in this region, are applied and evaluated in terms of its analytical and explanatory potential in this case. Special emphasis is placed on the evaluation of different types of anatomical quantification, and in the application of the Model of High and Low Potential for Survival, the conclusions stimulate future applications in similar feature sets. With respect to archaeological inferences derived from the analysis, it points out that the hunting of guanaco was made on a small scale, obtaining one or a few preys per event which were processed entirely. Differential anatomical representation and the frequency and variability of butchery marks, among other evidence, suggests differential transport of members, the intensive slaughter of the preys and the terminal position of Túnel I in the exploitation chain of this resource. However, also the dynamics of attritional processes, particularly the carnivore's action, would have had a significant impact on the differential preservation of elements.

KEY WORDS: Guanaco, Túnel I, Beagle Channel, zooarchaeology, taphonomy.

\section{INTRODUCCIÓN}

Uno de los rasgos distintivos de los conjuntos arqueofaunísticos de la región del canal Beagle es su diversidad. Desde las primeras ocupaciones correspondientes a poblaciones adaptadas al litoral marítimo, ca. 6.400 años AP, fue explotada con diferente intensidad una gran variedad de invertebrados -moluscos, crustáceos, decápodos y equinodermos- y diversos vertebrados -peces, aves, pinnípedos, cetáceos y camélidos(Orquera \& Piana, 1999; Piana et al. 2007; Tivoli, 2010; Zangrando, 2003, 2009). Según el modelo tradicionalmente propuesto para las poblaciones canoeras que habitaron la región, la caza de pinnípedos era la actividad fundamental de la subsistencia pues constituían la fuente básica de grasas y proteínas. Eran cazados principalmente en el medio acuático, desde la canoa y por medio de arpones con punta separable (Orquera \& Piana, 1999; Schiavini, 1993). Si bien sobre la base de evidencia paleoambiental, ecológica y arqueológica se sostuvo la estabilidad de este modelo a lo largo de 6 milenios, recientemente se ha propuesto la existencia de un proceso de intensificación hacia los últimos 1.500 años de la secuencia, con especial énfasis en la explotación de recursos de bajo rendimiento individual, específicamente peces y aves (Tivoli, 2012, 2014; Tivoli \& Zangrando, 2011; Zangrando, 2009, 2014).

Dentro de este panorama, los guanacos (Lama guanicoe) son concebidos como un recurso complementario, su obtención habría sido eventual, excepto en ciertas configuraciones ambientales donde se los pudiera hallar con relativa facilidad (Orquera \& Piana, 1996, 1999). Sus frecuencias en las arqueofaunas del canal Beagle son muy variables. Se registran conjuntos en los cuales se presenta como el taxón dominante: Componente Antiguo de Lancha Packewaia, Cuarto Componente de Túnel I (Orquera \& Piana, 1999), primer pulso de formación de Shamakush VIII (Piana \& Vázquez 2008), Isla El Salmón (Figuerero Torres \& Mengoni; Goñalons, 1986) y Shamakush I (Orquera \& Piana, 1996). Otros casos donde su incidencia es prácticamente nula: Ajej I (Piana et al. 2008), Túnel II (Piana \& Canale, 1995), Capa B de Imiwaia I y Túnel VII (Zangrando, 2009). También hay contextos donde sus frecuencias son intermedias en comparación con los otros taxones: capas K-L-M de Imiwaia I (Zangrando, 2009), Tercer y Quinto Componente de Túnel I (Orquera \& Piana, 1999) y Componentes Antiguo y Reciente de Mischiwen I. Esta información implica que la explotación de este recurso se mantuvo a lo largo de toda la secuencia, aunque no con la misma intensidad. En este sentido, en diversas oportunidades se han señalado fluctuaciones y tendencias temporales en el consumo de guanacos en la región (Orquera \& Piana, 1999, p. 115; Orquera, 2005, p. 113); particularmente Zangrando (2009, p. 86, 269 y $300)$ ha señalado un progresivo aumento en las frecuencias de sus restos en el lapso 5.000 y 1.000 años AP, pero también una notoria declinación en los conjuntos más tardíos.

En cuanto a las formas de obtención y aprovechamiento se ha propuesto que en términos generales las cacerías se habrían realizado a 


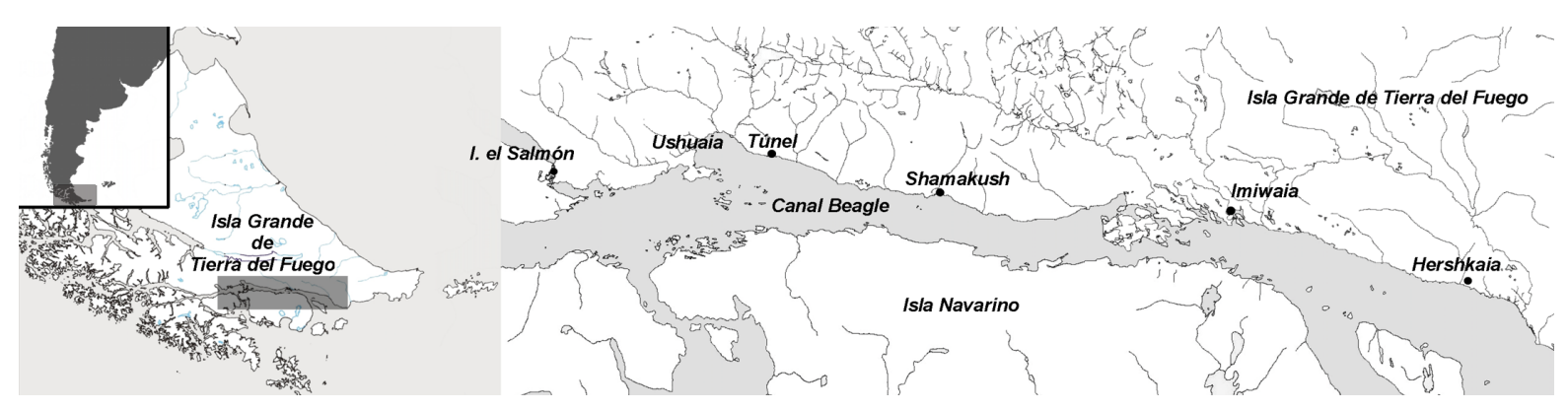

Fig. 1. Mapa de ubicación con localidades mencionadas en el texto.

cierta distancia de los campamentos, lo que implicaba mayores costos de transporte que los pinnípedos -que podrían ser remolcados hasta los campamentos en canoas- $y$ consecuentemente es esperable algún tipo de transporte diferencial de segmentos anatómicos a los campamentos residenciales (Orquera \& Piana, 1996, 1999, p. 109).

En el marco de las investigaciones desarrolladas por el Proyecto Arqueológico Canal Beagle, los estudios de subsistencia han tenido una notoria gravitación desde sus inicios. Sin duda, estos estudios han contribuido de manera significativa al desarrollo de la arqueología regional, habiendo propuesto modelizaciones del sistema de subsistencia de suma relevancia. Se destaca el caso de los pinnípedos (Schiavini, 1993), los peces (Zangrando, 2003, 2009), las aves (Tivoli, 2010) y los moluscos (Orquera \& Piana, 2000, 2001). Además, recientes análisis han focalizado sus esfuerzos en el estudio de las tendencias temporales de estas pautas (Tivoli, 2010; Tivoli \& Zangrando, 2011; Zangrando, 2009). Sin embargo, en la arqueología del sur de Tierra del Fuego solo recientemente los estudios arqueofaunísticos han focalizado suatención sobre los guanacos como recurso. Tal es el caso de los niveles inferiores de Imiwaia I y los conjuntos de Heshkaia (Punta Moat) (Alunni, 2013; Alunni \& Zangrando, 2012), en donde se abordan temáticas relativas a la representación anatómica y modificaciones sobre las superficies óseas, entre otros aspectos. Aunque por el momento la restricción de estos análisis a escasos conjuntos limita sensiblemente el potencial de las discusiones al respecto. En este contexto, el análisis del subconjunto de restos de guanaco de la capa $\mathrm{D}$ de Túnel I resulta relevante en al menos dos sentidos: en la escala intra-regional apunta a establecer modos de explotación para este contexto espacio-temporal. A escala interregional permitirá contar con datos tafonómicos e inferencias comportamentales comparables con contextos ambientales y sociales muy diferentes donde también se explotaron guanacos (Borrero, 1990; Mengoni Goñalons, 1999; Muñoz, 2005, 2008; Santiago, 2013; Santiago \& Vázquez, 2012, entre otros). Por otro lado, en la región del canal Beagle son escasos los contextos con información tafonómica detallada. Si bien asumimos que las acumulaciones de conchal que componen los sitios son de naturaleza eminentemente antrópica, también se hace evidente que los procesos de formación actuantes son de por sí muy complejos y variables. En este sentido es que consideramos que los análisis tafonómicos deben ser incluidos en las agendas de investigación, en conjunción con los esfuerzos por delinear las estrategias económicas implementadas en el pasado (Borrero, 2007b).

\section{TÚNEL I: EL YACIMIENTO Y EL SEGUNDO COMPONENTE}

La localidad arqueológica Túnel se ubica sobre la costa norte del canal Beagle a unos $10 \mathrm{~km}$ al este de Ushuaia (Fig. 1). Alli se encuentra emplazada más de una docena de sitios arqueológicos, de los que han sido excavados cuatro: Túnel I, II, VII y XIV (Estévez \& Vila, 1995; Orquera \& Piana, 1988, 1999; Piana, 1984; Piana \& Canale, 1995). Túnel I es un conchero de importantes dimensiones asentado sobre un talud rocoso a unos $80 \mathrm{~m}$ de la costa actual y a 15 msnm. Los trabajos de excavación fueron realizados entre los años 1976 y 1986, alcanzando una superficie de $150 \mathrm{~m}^{2}$ y un volumen de $152 \mathrm{~m}^{3}$. Fueron discriminadas seis capas arqueológicamente fértiles, denominadas de la superior a la inferior "A" a " $F$ " y varias lentes 
menores; en la mayoría de los casos se trata de capas de conchero de naturaleza antrópica. Sobre la base de las características del instrumental recuperado y la cronología, los hallazgos fueron agrupados en seis componentes arqueológicos (Orquera \& Piana, 1988, 1992, 1999; Piana, 1984).

El Segundo Componente, el más voluminoso y el que mayor proporción de restos contenía, incluye las Capas F Superior, E y D, está compuesto por una sucesión de numerosas ocupaciones fechadas entre 6.400 y 4.500 años AP (ver Orquera \& Piana, 1999. Figs. 14 y 15 y Cuadro II). Los conjuntos arqueofaunístico y artefactual presentan tendencias relativamente estables que justificaron considerarlo como una unidad pese a su volumen y extensión temporal, se recuperaron más de 126.000 especímenes óseos, con una clara preponderancia de pinnípedos (Orquera \& Piana, 1988, 1999). La mayor parte del volumen y de los restos de este componente, provienen de la capa D: $99 \%$ de los objetos ornamentales, $66 \%$ del instrumental y $85 \%$ de la fauna. Las principales tendencias artefactuales y faunísticas indican la especialización en la explotación de recursos de origen marino. Esta especialización se manifiesta, además de la importancia relativa de los pinnípedos y otros recursos litorales, en el énfasis en la confección de instrumentos y ornamentos sobre materias primas de origen marino (p. e. huesos de cetáceos y valvas) $y$ en la cantidad de instrumentos especializados en su obtención, como los distintos tipos de arpones. El conjunto de instrumentos líticos se caracteriza por su poca especialización morfológica (Álvarez, 2004; Orquera \& Piana, 1988, 1999: cuadros VI y VII).

En cuanto a los valores de abundancia taxonómica de vertebrados en el Segundo Componente de Túnel I, el taxón mejor representado es pinnípedos, alcanzando un $63 \%$ del NISP e incluyendo dos especies: Arctocephalus australis y Otaria flavescens (Tabla 1), de los cuales 59.283 especimenes $(74,10 \%)$ provienen de la Capa D (Orquera com. pers.).

De los restos de pinnípedos los más abundantes corresponden a A. australis: -NMI 365 (Orquera com. pers.), frente a solo nueve de $O$. flavescens. Entre los primeros se verificó una tendencia hacia la caza de machos (aproximadamente el $80 \%$ de los especímenes con sexo determinado) y una alta proporción de ejemplares juveniles. En cuanto a la estacionalidad, los resultados denotan una mayor incidencia en la explotación en los meses invernales: el $90 \%$ de los casos habrían sido cazados entre marzo y septiembre (Orquera \& Piana, 1999; Schiavini, 1993).

Los restos de aves alcanzan el 29\% del NISP, incluyendo una importante variedad de especies, aunque los taxones mejor representados son los cormoranes (Phalacrocórax sp.) y los pingüinos (Familia Sphenicidae) (Piana et. al. 2007). Los restos de guanacos aparecen en tercer lugar, sin embargo su tamaño corporal respecto de las aves lo ubican en segundo lugar de aporte a la dieta. Los cetáceos representan menos del 1\% del NISP $y$ se registran diversas especies de delfinidos y cetáceos mayores. Entre los peces, los taxa mejor representados corresponden a nototénidos pequeños (Paranotothenia magellanica y Patagonotothen sp.) y a sardinas (Clupeidae sp.) (Zangrando, 2009).

Tabla 1. NISP y NISP\% del Segundo Componente (tomado de Orquera \& Piana, 1999: cuadro X).

\begin{tabular}{|c|c|c|}
\hline Taxón & NISP & \%NISP \\
\hline Pinnípedos & 80.000 & 63,48 \\
\hline Aves & 36.057 & 28,61 \\
\hline Guanacos & 6.482 & 5,14 \\
\hline Peces & 2.611 & 2,08 \\
\hline Cetáceos & 858 & 0,69 \\
\hline Total & 126.008 & 100 \\
\hline
\end{tabular}

Si bien la definición del Segundo Componente incluye las capas F Superior, E y D (Orquera \& Piana, 1988), en este trabajo se analizan los restos de guanaco correspondientes solo a la capa D, los cuales incluyen el 90,3\% del total de los restos de este taxón asignados al Segundo Componente. Las razones de este recorte se deben fundamentalmente a tres condiciones. Primero: el conjunto recuperado de la capa $\mathrm{D}$ incluye un número absoluto y un porcentaje de especímenes suficiente para considerarlo representativo del total del Segundo Componente. Segundo: aunque 
por el momento no se dispone de fechados correspondientes a la capa F Superior, las fechas máximas obtenidas para la capa $\mathrm{E}$ son equivalentes o incluso más modernas que los fechados más antiguos obtenidos para la capa $\mathrm{D}$, por lo cual la inclusión de sus materiales en este estudio no garantiza la ampliación de su rango temporal. Por último, y posiblemente más importante para el enfoque tafonómico aquí adoptado, es la naturaleza de estas capas. La capa D corresponde a una capa de conchero compuesta en gran proporción por restos de valvas de moluscos (mayoritariamente Mitylus sp.), en cambio las unidades E y F corresponden a capas de tierra. Estas diferencias hacen que el potencial de los procesos diagenéticos actuantes y las condiciones de conservación dentro de estas capas sea diferente $\mathrm{y}$, por ende, que los restos óseos contenidos en ellas hayan estado sometidos a diferentes agentes o al accionar de procesos similares pero muy variables en intensidad. Considero que, sin perjuicio de la unicidad cultural del conjunto definido como Segundo Componente, para este análisis es más adecuada la exclusión de los especímenes recuperados en las capas E y F. $\mathrm{Su}$ eventual análisis tafonómico por separado y su posterior comparación y calibración de los procesos actuantes, en parte tomando como punto de comparación los datos obtenidos para la capa $\mathrm{D}$, constituye indudablemente un interesante desafío para futuros análisis.

\section{ANÁLISIS ZOOARQUELÓGICO Y TAFONÓMICO DE LOS RESTOS DE GUANACO}

\section{Perfil de mortalidad}

Por razones de espacio en esta ocasión solo se presentan sucintamente algunos datos que serán relevantes en la interpretación de las pautas de explotación de las presas. Los datos aquí mencionados son producto de la determinación de edad a partir de las series molares mandibulares completas o casi completas. Dado el nivel de fragmentación solo fue posible determinar la edad ajustada a lapsos menores al año en 17 individuos.

Respecto a los resultados obtenidos hay algunos aspectos a destacar: en primer lugar todo parece indicar que se seleccionaron preferentemente presas en edad reproductiva; la mayoría de ellas tenían entre 3 y 6 años de edad al momento de su muerte ( 8 individuos de los cuales cinco de ellos tenían 3 años), lo que concuerda con un modelo del tipo prime dominated (Stiner, 1991). También se registraron cuatro animales subadultos de entre 1 y 2 años, dos chulengos menores de 1 año y tres individuos seniles mayores de 6 años. Dentro de este perfil, debemos destacar la identificación de restos de crías de pocos meses de edad -dos individuos de menos de 6 meses-, a los cuales se suman otros restos postcraneales de animales muy pequeños que podrían corresponder a los casos determinados por mandíbulas o bien a otros animales de este rango etario. La presencia de individuos tan jóvenes tiene dos implicancias importantes: por un lado son indicadores confiables de cacerías realizadas en verano u otoño y por otro que, al menos en algunas oportunidades, fueron explotadas tropillas familiares con hembras y crías, aunque también es probable que los individuos mayores de 6 años se hubiesen comportado como individuos aislados y no como miembros de tropillas estables. Lamentablemente, el grado de fragmentación de las pelvis no ha permitido efectuar determinaciones de sexo seguras en función de su morfología. Futuros análisis implementando otras metodologías (tamaño y morfología de caninos y morfometría pélvica) podrán clarificar esta cuestión.

\section{Abundancia anatómica}

El NISP de guanaco incluye especímenes -generalmente fragmentos pequeños- que no fueron asignados a categorías anatómicas precisas, por ejemplo: vértebras no determinadas, dientes no determinados y sobre todo fragmentos de diáfisis de huesos largos. Dado que la región del Beagle no fue habitada por otros artiodáctilos hasta la introducción de la ganadería de origen europeo y que las características estructurales y anatómicas de los huesos de pinnípedos -únicos animales de tamaño comparable presentes en los conjuntosson muy diferentes a las de los guanacos, considero confiable la inclusión de estos especímenes en esta cuantificación pese a la ausencia de otros rasgos diagnósticos.

Durante los trabajos de clasificación anatómica se puso especial énfasis en la 
identificación de los fragmentos diafisiarios. Como material comparativo se utilizaron esqueletos de referencia de distintas edades, con el objetivo de incrementar el potencial de determinación de estas porciones anatómicas. La asignación de fragmentos diafisarios a categorías anatómicas específicas estuvo basada sobre la identificación de rasgos unívocos, como inserciones musculares (Mengoni Goñalons, 1999) o en algunos casos la forma de la sección trasversal. En ciertas ocasiones la asignación provisoria de algunos fragmentos a elementos específicos permitió realizar remontajes, asistiendo de esta manera a su determinación definitiva. La inclusión del máximo posible de fragmentos de diáfisis, tuvo por meta subsanar los problemas metodológicos advertidos por diversos autores, vinculados a la cuantificación de huesos largos basada en los extremos proximales y distales (Lyman, 1994; Marean, 1995; Marean \& Cleghorn, 2003; Marean \& Frey, 1997; Marean \& Spencer, 1991; entre otros).

El NMI calculado para el conjunto es 23 y fue estimado a partir de la representación de la diáfisis del radiocúbito derecho, asimismo esta porción anatómica alcanza los valores más altos de MAU y \% de Supervivencia de todo el conjunto. Aunque puede verificarse la presencia de todas las regiones anatómicas, estas están representadas en frecuencias muy variables (Tabla 2 y Fig. 2). Los valores más altos corresponden al esqueleto apendicular y mandíbulas, los primeros representados fundamentalmente por las diáfisis de húmeros, radiocúbitos, tibias y el calcáneo. Otra tendencia clara es la sub-representación de los extremos proximales y distales de los huesos largos en relación con frecuencias mucho más altas de regiones diafisiarias. Las regiones menos representadas corresponden mayoritariamente al tórax y la columna vertebral, rondando estimaciones cercanas al $50 \%$ del índice de supervivencia o del MAU\%.

El patrón general que surge del análisis sugiere una mayor frecuencia de descarte en el sitio de huesos del esqueleto apendicular con relación al axial, lo que podría indicar una selección hacia segmentos con alto rendimiento de carne y médula, o bien una mayor frecuencia de segmentos anatómicos más fácilmente transportables una vez realizado el trozamiento primario de las carcasas, como son los miembros completos. Sin embargo, las bajas frecuencias de algunos elementos o de sus porciones de baja densidad ósea podrían indicar

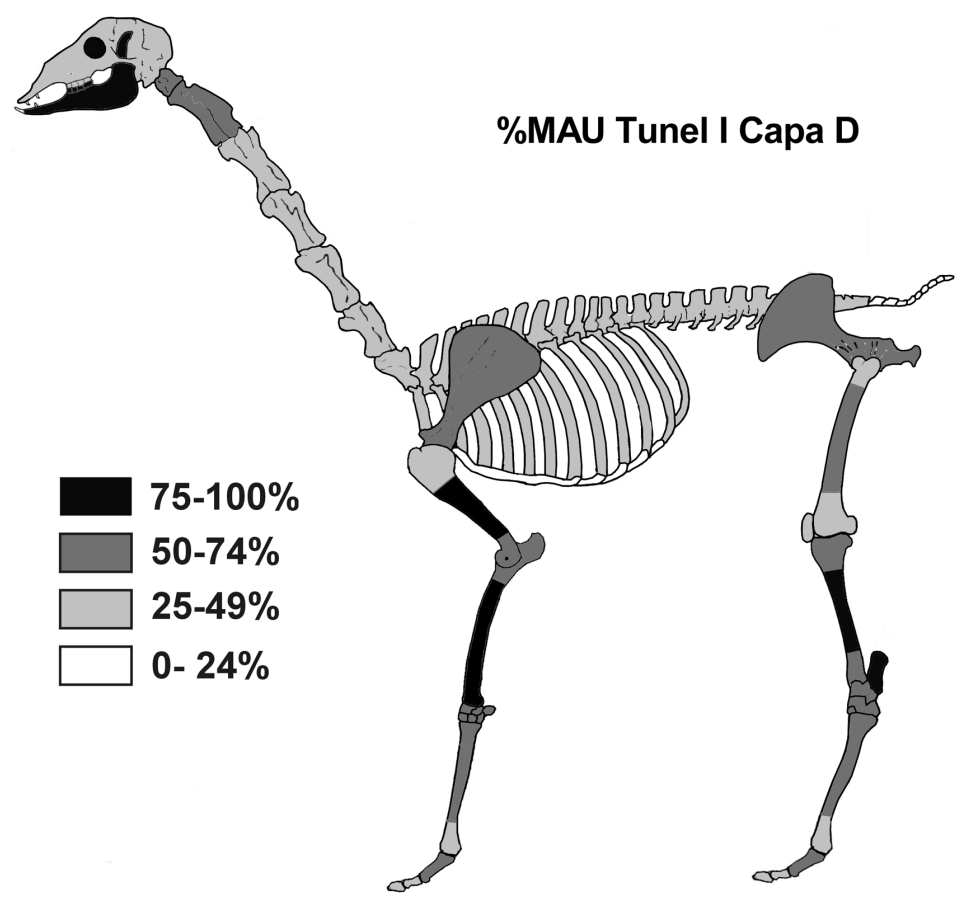

Fig. 2. Representación de MAU\% Túnel I Capa D. 
la acción de procesos atricionales relativamente intensos que habrían afectado la preservación del conjunto.

Algunos elementos del autopodio, no asociados con masas musculares grandes, también presentan frecuencias medias o altas (p. e. falanges y metapodios), estos podrían haber sido introducidos al sitio aun articulados en unidades mayores y adheridos al cuero: riders en el sentido de Binford (1981). Lo cual sería coherente con la hipótesis del transporte selectivo de los miembros completos como unidades primarias. Aunque para el caso de los metapodios también es posible argumentar su potencial utilización como materia prima o su selección por el alto contenido de ácidos oleicos en su grasa medular (Miotti \& Marchionni, 2014). En los conjuntos de la región, y el Segundo Componente de Túnel I no es la excepción, es común hallar diversos instrumentos confeccionados sobre sus diáfisis. En consecuencia el empleo de los metapodios como materia prima, así como del cuero y los tendones asociados o su contenido graso podrían dar cuenta del patrón registrado.

La relación NISP/NME brinda una estimación del grado de fragmentación de la muestra (Lyman, 1994, p. 336-338), en este caso los índices resultantes indican una considerable fragmentación de los huesos, con valores promedio que rondan entre 2 y 4 (Tabla 2). Los índices muy altos se dan en aquellos elementos más susceptibles a la fragmentación: cráneo, mandíbula y pelvis $y$, por el contrario, valores iguales o cercanos a 1 en elementos o porciones muy pequeños y de alta densidad: carpos, tarsos, metatarso proximal y tibia distal. En los huesos largos el índice de fragmentación es mayor para las diáfisis que para los extremos proximales y distales.

Ahora bien, ¿el perfil anatómico descripto podría estar influido por los procedimientos de determinación y cuantificación empleados para el esqueleto axial? En otras palabras, ¿hasta qué punto la baja representación del esqueleto axial en comparación del apendicular es un artificio metodológico dependiente de los procedimientos de cuantificación diferenciales para estas regiones? Este razonamiento se basa en la simple revisión de los procedimientos comúnmente empleados para la cuantificación de vértebras y costillas, en comparación con los aplicados a los huesos pares de los miembros o a elementos únicos, como el atlas, el axis o la mandíbula.

Como es de uso común en el cálculo del MAU, los NME en huesos pares fueron divididos por 2 y para categorías equivalentes a las regiones cervical, toráxica, lumbar y costal, por la frecuencia de elementos por porción anatómica: 5, 12, 7 y 24 respectivamente. Es posible que tales procedimientos impliquen la subrepresentación del sector axial frente al método de cuantificación de huesos pares con lateralidad determinable; en consecuencia, brindar un panorama sesgado de los patrones de selección y/o conservación diferencial de los conjuntos. Por ejemplo, en el caso que aquí nos ocupa, como en muchos otros, las $1^{\text {ra }}$ y $2^{\text {da }}$ vértebras cervicales -atlas y axis- suelen estar mejor representadas que el resto del segmento cervical (ver Tabla 2 y Fig. 2), posiblemente muchos de estos casos estén relacionados, al menos en parte, con el método de cuantificación empleado para estas vértebras en contraposición con el resto de la región cervical indiferenciada. La asimetría en los procedimientos de cuantificación entre esqueleto axial y apendicular se incrementaría más aun en los casos en que los criterios de identificación de los fragmentos de diáfisis se vean mejorados. Considero que esta problemática es particularmente significativa, ya que las bajas frecuencias del esqueleto axial registrada en diversos contextos ha reforzado en muchos casos las hipótesis de la alta incidencia de destrucción in situ de los conjuntos (Lyman, 1992; Marean \& Frey, 1997).

A fin de morigerar este efecto se utilizaron otros elementos bien identificables del esqueleto axial -ya sea como elementos completos o por alguna de sus porciones- y se procedió a su determinación y cuantificación como elementos únicos y diferenciados, extrapolando luego tal cuantificación a toda la región anatómica. Los huesos utilizados fueron: para la columna vertebral, además de los ya mencionados atlas y axis, la $7^{\text {ma }}$ vértebra cervical; para la región toráxica la $1^{\text {ra }}$ vértebra y para la lumbar la $7^{\mathrm{ma}}$ vértebra. Para el caso de la región costal se identificaron la primera y segunda costilla, a las cuales en la mayoría de los casos puede asignarse su lateralidad y ser cuantificada como un hueso par. A través de la aplicación de esta forma de cálculo se esperaba arribar a estimaciones del esqueleto axial más 
Tabla 2. Cuantificación de restos óseos de guanaco. Capa D de Túnel I.

\begin{tabular}{|c|c|c|c|c|c|c|c|c|c|c|c|c|c|c|c|}
\hline \multicolumn{2}{|c|}{ Unidad Anatómica. } & 离 & 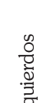 & 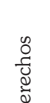 & $\begin{array}{l}\stackrel{0}{0} \\
\stackrel{0}{0} \\
\stackrel{\Xi}{\Xi}\end{array}$ & $\begin{array}{l}\text { 퓨 } \\
\text { के } \\
\text { के }\end{array}$ & 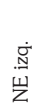 & $\begin{array}{l}\stackrel{\ddot{g}}{0} \\
\text { 㞱 }\end{array}$ & $\begin{array}{l}\text { 즁 } \\
\text { 岁 }\end{array}$ & $\sum_{i=1}^{\omega}$ & $\sum_{\Sigma}$ & 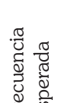 & 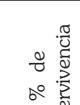 & 导 & 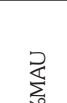 \\
\hline \multirow{4}{*}{ Cabeza } & Cráneo & 29 & 47 & 61 & 34 & 171 & & & 10 & 17.1 & 10 & 23 & 43.47 & 10 & 47.61 \\
\hline & Dientes craneales & & 12 & 6 & 1 & 19 & & & & & & & & & \\
\hline & Mandíbula & 4 & 49 & 49 & 28 & 130 & 18 & 13 & 18 & 7.22 & 18 & 23 & 78.26 & 18 & 85.71 \\
\hline & Dientes mandibulares & & 29 & 23 & 4 & 56 & & & & & & & & & \\
\hline \multirow{9}{*}{ Esqueleto Axial } & Vert. Atlas & 17 & & & & 17 & & & 11 & 1.54 & 11 & 23 & 47.82 & 11 & 52.38 \\
\hline & Vert. Axis & 18 & & & & 18 & & & 11 & 1.63 & 11 & 23 & 47.82 & 11 & 52.38 \\
\hline & Vert. Cervical & 193 & & & & 193 & & & 50 & 3.86 & 10 & 115 & 43.47 & 10 & 47.61 \\
\hline & Vert. Torácica & 150 & & & & 150 & & & 69 & 2.17 & 6 & 276 & 25 & 5.75 & 27.38 \\
\hline & Vert. Lumbar & 237 & & & & 237 & & & 57 & 4.15 & 8 & 161 & 35.40 & 8.14 & 38.76 \\
\hline & Sacro & 21 & & & & 21 & & & 7 & 3.00 & 7 & 23 & 30.43 & 7 & 33.33 \\
\hline & Vert. caudal & & & & 27 & 27 & & & & & & & & & \\
\hline & Costilla & & 105 & 110 & 236 & 451 & 65 & 77 & 142 & 3.17 & 6 & 552 & 25.72 & 5.91 & 28.14 \\
\hline & Esternebra & 7 & & & & 7 & & & & & & & & & \\
\hline \multirow{15}{*}{ Miembro Anterior } & Escápula & & 27 & 40 & 25 & 92 & 10 & 14 & 24 & 3.83 & 14 & 46 & 52.17 & 12 & 57.14 \\
\hline & Húmero $\mathrm{px}$ & & 13 & 12 & 11 & 36 & 7 & 8 & 15 & 2.4 & 8 & 46 & 32.60 & 7.5 & 35.71 \\
\hline & Húmero diáfisis & & 52 & 50 & 5 & 107 & 16 & 16 & 32 & 3.34 & 16 & 46 & 69.56 & 16 & 76.19 \\
\hline & Húmero ds & & 26 & 17 & 7 & 50 & 15 & 10 & 25 & 2.00 & 15 & 46 & 54.34 & 12.5 & 59.52 \\
\hline & Radiocúb. px & & 16 & 60 & 6 & 82 & 11 & 14 & 25 & 3.28 & 14 & 46 & 54.34 & 12.5 & 59.52 \\
\hline & Radiocúb. df & & 66 & 62 & 20 & 148 & 19 & 23 & 42 & 3.52 & 23 & 46 & 91.30 & 21 & 100 \\
\hline & Radiocúb. ds & & 39 & 42 & 0 & 81 & 19 & 19 & 38 & 2.13 & 19 & 46 & 82.60 & 19 & 90.47 \\
\hline & Cuneiforme & & 10 & 11 & & 21 & 10 & 11 & 21 & 1.00 & 11 & 46 & 45.65 & 10.5 & 50 \\
\hline & Escafoide & & 10 & 10 & & 20 & 10 & 10 & 20 & 1.00 & 10 & 46 & 43.47 & 10 & 47.61 \\
\hline & Lunar & & 11 & 14 & & 25 & 11 & 14 & 25 & 1.00 & 14 & 46 & 54.34 & 12.5 & 59.52 \\
\hline & Magnum & & 12 & 9 & & 21 & 12 & 9 & 21 & 1.00 & 12 & 46 & 45.65 & 10.5 & 50 \\
\hline & Pisciforme (acces) & & 8 & 11 & & 19 & 8 & 11 & 19 & 1.00 & 11 & 46 & 41.30 & 9.5 & 45.23 \\
\hline & Trapezoide & & 4 & 4 & & 8 & 4 & 4 & 8 & 1.00 & 4 & 46 & 17.39 & 4 & 19.04 \\
\hline & Unciforme & & 9 & 11 & & 20 & 9 & 11 & 20 & 1.00 & 11 & 46 & 43.47 & 10 & 47.61 \\
\hline & Metacarpo px & & 28 & 23 & 5 & 56 & 11 & 11 & 22 & 2.54 & 11 & 46 & 47.82 & 11 & 52.38 \\
\hline \multirow{17}{*}{ Miembro Posterior } & Pelvis & 3 & 61 & 49 & 30 & 143 & 12 & 14 & 14 & 10.21 & 14 & 23 & 60.86 & 14 & 66.66 \\
\hline & Fémur px & & 21 & 20 & 2 & 43 & 11 & 9 & 20 & 2.15 & 11 & 46 & 43.47 & 10 & 47.61 \\
\hline & Fémur diáfisis & & 46 & 54 & 7 & 107 & 15 & 17 & 31 & 3.45 & 17 & 46 & 67.39 & 15.5 & 73.80 \\
\hline & Fémur ds & & 13 & 28 & 7 & 48 & 5 & 15 & 20 & 2.40 & 15 & 46 & 43.47 & 10 & 47.61 \\
\hline & Rótula & & 10 & 8 & 2 & 20 & 9 & 8 & 17 & 1.17 & 9 & 46 & 36.95 & 8.5 & 40.47 \\
\hline & Tibia px & & 26 & 32 & 8 & 66 & 11 & 14 & 25 & 2.64 & 14 & 46 & 54.34 & 12.5 & 59.52 \\
\hline & Tibia diáfisis & & 46 & 55 & 6 & 107 & 14 & 19 & 33 & 3.24 & 19 & 46 & 71.73 & 16.5 & 78.57 \\
\hline & Tibia ds & & 22 & 16 & & 38 & 15 & 11 & 26 & 1.46 & 15 & 46 & 56.52 & 13 & 61.90 \\
\hline & Astrágalo & & 17 & 12 & & 29 & 15 & 11 & 26 & 1.11 & 15 & 46 & 56.52 & 13 & 61.90 \\
\hline & Calcaneo & & 36 & 42 & 1 & 79 & 18 & 18 & 36 & 2.19 & 18 & 46 & 78.26 & 18 & 85.71 \\
\hline & $1^{\circ}$ Tarsiano & & 1 & 2 & & 3 & 1 & 2 & 3 & 1.00 & 2 & 46 & 6.52 & 1.5 & 7.14 \\
\hline & Cuboide & & 10 & 14 & & 24 & 10 & 14 & 24 & 1.00 & 14 & 46 & 52.17 & 12 & 57.14 \\
\hline & Endocuneiforme & & 5 & 15 & & 20 & 5 & 15 & 20 & 1.00 & 15 & 46 & 43.47 & 10 & 47.61 \\
\hline & Navicular & & 9 & 15 & & 24 & 9 & 15 & 24 & 1.00 & 15 & 46 & 52.17 & 12 & 57.14 \\
\hline & Fíbular & & 10 & 10 & & 20 & 10 & 10 & 20 & 1.00 & 10 & 46 & 43.47 & 10 & 47.61 \\
\hline & Sesamoideos & & & & 21 & 21 & & & 21 & 1.00 & & 46 & & & \\
\hline & Metatarso px & & 26 & 29 & 3 & 58 & 16 & 15 & 31 & 1.87 & 16 & 46 & 67.39 & 15.5 & 73.80 \\
\hline \multirow{5}{*}{$\begin{array}{c}\text { Autopodios } \\
\text { indiferenciados }\end{array}$} & Metapodio df & & & & 192 & 192 & & & 50 & 3.84 & 13 & 92 & 54.34 & 12.5 & 59.52 \\
\hline & Metapodio ds & & & & 125 & 125 & & & 37 & 3.37 & 10 & 92 & 40.21 & 9.25 & 44.04 \\
\hline & $1^{\circ}$ Falange & & & & 279 & 279 & & & 107 & 2.60 & 14 & 184 & 58.15 & 13.37 & 63.66 \\
\hline & $2^{\circ}$ Falange & & & & 146 & 146 & & & 75 & 1.94 & 10 & 184 & 40.70 & 9.37 & 44.61 \\
\hline & $3^{\circ}$ Falange & & & & 51 & 51 & & & 49 & 1.04 & 7 & 184 & 26.63 & 6.12 & 29.14 \\
\hline \multirow{5}{*}{ Otros } & Dient nd (mol/premol) & & & & 58 & 58 & & & & & & & & & \\
\hline & Dient nd (caninos) & & & & 10 & 10 & & & & & & & & & \\
\hline & Vert ND & & & & 41 & 41 & & & & & & & & & \\
\hline & H. Largo & & & & 1725 & 1725 & & & & & & & & & \\
\hline & Guanac ND & & & & 94 & 94 & & & & & & & & & \\
\hline \multicolumn{2}{|c|}{ NISP Total } & & & & & 5854 & & & 1441 & 4.06 & & & & & \\
\hline
\end{tabular}


comparables con las obtenidas para el apendicular.

Los resultados obtenidos y su comparación con los valores calculados mediante el método tradicional se consignan en la Tabla 3. Como puede observarse, se registró un incremento en todos los casos. Si bien las diferencias resultaron de menor cuantía que las que se esperaba a priori, tanto en costillas como en vértebras toráxicas los incrementos no son despreciables, alcanzado respectivamente valores de 7,57 y 10,71 del \%MAU, en comparación con las estimaciones iniciales. Sin embargo, los resultados obtenidos con este procedimiento no alcanzan a revertir las tendencias generales que surgen de las estimaciones previas: la representación del esqueleto axial se incrementa, pero sigue siendo minoritaria con relación al apendicular.

Selección vs. Preservación:

carne, grasa y huesos

Dada la representación diferencial de elementos establecida, se exploraron las relaciones entre el \%MAU, y algunos de los marcos de referencia disponibles para guanaco, utilizando el test de Spearman. Las correlaciones entre la abundancia de partes esqueletarias y los índices de rendimiento económico persiguen la identificación de patrones de selección y descarte (Binford, 1978, 1981; Lyman, 1994, entre muchos otros). Por otro lado, la evaluación de posibles sesgos en la representación de partes en función de su potencial de supervivencia es evaluada correlacionando los valores de MAU con los de densidad mineral ósea. Fueron utilizados en conjunto tres de los índices de rendimiento económico disponibles para guanaco: Índice de Utilidad de Carne (Meat Utility Index, MUI) y Indice Estandarizado de Carne y Médula (Standarized Meat + Marrow Index, SM+MI) propuestos por Borrero (1990), además del Índice de Utilidad General Modificado (Modified General Utility Index, MGUI) modificado de los valores anteriores por Lyman (1992, 1994). Para la correlación con los valores de Densidad Mineral Ósea (DMO) se utilizaron las estimaciones propuestas por Elkin (1995).

Se realizó una batería de tests poniendo en práctica distintas combinaciones de cuantificaciones de huesos largos a fin de explorar las variaciones en los resultados con distintos tipos de estimaciones. Para el esqueleto axial en todos los casos se utilizaron los valores calculados con la aplicación de criterios de mayor distinción anatómica consignados en Tabla 3. En primera instancia se utilizaron las estimaciones tradicionales, basadas sobre los valores de los extremos proximales y distales de los principales huesos largos (húmero, radiocúbito, fémur y tibia), excluyendo los valores calculados para las diáfisis (Fig. 3 y Tabla 4). Este análisis permitió utilizar directamente las estimaciones publicadas de los índices de utilidad, los cuales consignan valores discriminados para los extremos proximales y distales, sin considerar las diáfisis o los huesos completos como unidad

Tabla 3. Estimación de valores de abundancia anatómica en esqueleto axial, a la izquierda las estimaciones basadas sobre método tradicional y a la derecha según criterios de mayor distinción anatómica.

\begin{tabular}{|c|c|c|c|c|c|c|}
\hline & \multicolumn{2}{|c|}{ Índice de supervivencia } & \multicolumn{2}{|c|}{ MAU } & \multicolumn{2}{c|}{ \%MAU } \\
\hline Vert. Cervical & 43,47 & 47,82 & 10 & 11 & 47,61 & 52,38 \\
\hline Vert. Torácica & 25 & 34,78 & 5.75 & 8 & 27,38 & 38,09 \\
\hline Vert. Lumbar & 35,4 & 39,13 & 8.14 & 9 & 38,76 & 42,85 \\
\hline Costilla & 25,72 & 32,6 & 5.91 & 7.5 & 28,14 & 35,71 \\
\hline
\end{tabular}

Tabla 4. Resultados de correlación $r$ de Spearman con índices de rendimiento económico y densidad mineral ósea y el MAU calculado a partir de extremos proximales y distales en huesos largos.

\begin{tabular}{|c|c|c|c|c|}
\hline & $\mathrm{N}^{\circ}$ de pares & $\mathrm{r}$ Spearman & $\mathrm{P}$ & \\
\hline MUI vs. MAU & 27 & -0.4139 & 0.0319 & significativa \\
\hline SM+MI vs. MAU & 27 & -0.01319 & 0.9480 & no significativa \\
\hline MGUI vs. MAU & 27 & -0.2533 & 0.2023 & no significativa \\
\hline DMO vs. MAU & 28 & 0.7727 & $<0.0001$ & muy significativa \\
\hline
\end{tabular}



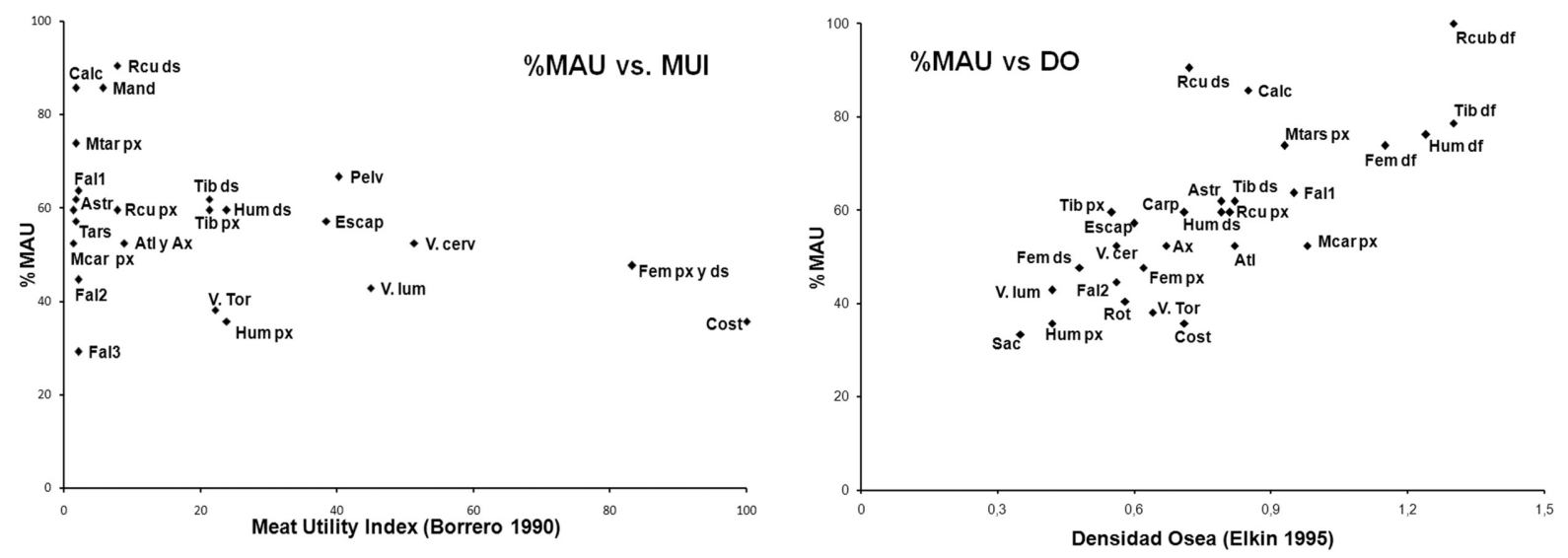

Fig. 3. Izquierda gráfico de la correlación entre Meat Utility Index y \%MAU, derecha gráfico de la correlación entre DMO y \%MAU.

analítica (Borrero, 1990; Lyman, 1992).

Los resultados de las correlaciones con índices de rendimiento (\%MAU vs. MUI, SM+MI, MGUI) (Tabla 4) presentan una tendencia general negativa y de ellos sólo la correlación con el Meat Utility Index (Borrero, 1990) puede considerarse significativa, aunque no exhibe un alto grado de correlación entre las dos variables. Estos resultados sugieren que los elementos descartados en el sitio no habrían sido seleccionados por su rendimiento en carne y/o grasa $y$, en caso de haber alguna tendencia, esta implicaría el descarte de elementos de rendimiento bajo y medio.

Por otro lado, la correlación con la DMO sí arrojó resultados altamente significativos (Tabla 4 y Fig. 3) ${ }^{1}$. El alto grado de correlación entre ambas variables indica una mejor representación de elementos de alta densidad mineral y menores frecuencias de elementos con bajas densidades. En primera instancia esto apunta a la acción de procesos atricionales como causa de la destrucción de especímenes con bajas expectativas de supervivencia. Esta combinación de correlaciones (positiva y significativa entre MAU y DMO y no significativa entre MAU y rendimiento económico) ubica este conjunto en la Clase 4 de Lyman (1994) en la cual la destrucción y pérdida de elementos habría tenido una importante gravitación en la conformación del conjunto. La combinación alternativa, brindada por la correlación negativa entre el \%MAU y el MUI y positiva entre el MAU y

1 Para este caso se utilizaron las estimaciones que incluyen las diáfisis de los huesos largos consignadas en la Tabla 3, ya que los datos relativos a huesos largos publicados por la DMO ubica el conjunto en la Clase 1 del modelo de Lyman, para la cual se plantean problemas de equifinalidad, siendo dificultoso discernir entre el descarte de huesos de bajo rédito y la pérdida de porciones de baja densidad mineral, como causales de la conformación del conjunto. La razón fundamental de estos problemas de equifinalidad radica en la correlación negativa y altamente significativa entre los índices económicos y los de DMO que se comprueba en el guanaco (ver discusión en Lyman, 1994, p. 258-259).

Desde hace varios años numerosos estudios han demostrado claramente las limitaciones de las estimaciones de huesos largos realizadas sobre la base del conteo de los extremos proximales y distales, resaltando la necesidad de incluir los fragmentos de diáfisis en su cuantificación (Bunn \& Kroll, 1986; Cleghorn \& Marean, 2004; Marean, 1995; Marean \& Frey, 1997; Marean \& Spencer, 1991). En este sentido y como segunda instancia analítica se utilizaron las estimaciones de abundancia de huesos largos efectuadas a partir de los fragmentos diafisarios, en todos los casos mayores que las estimaciones de los extremos proximales y distales (Tabla 2). En otras palabras, se incluyeron todos los elementos y porciones anatómicas, con la diferencia respecto de las correlaciones previas, que los huesos largos fueron tabulados como elementos completos. Esto implicó la adaptación de las medidas de rendimiento económico disponibles, ya que estas consignan

Elkin (1995) discriminan entre los valores de densidad determinados para las porciones distal, proximal $y$ diáfisis 
Tabla 5. Resultados de correlación $r$ de Spearman con índices de rendimiento económico y el MAU calculado a partir de los valores de diáfisis de huesos largos. $\mathrm{SM}+\mathrm{MI}$ (máx.) * = valores máximos entre distal y proximal. SM+MI (prom.) ${ }^{* *}=$ valores promediados entre el valor consignado para el extremo proximal y el distal.

\begin{tabular}{|c|c|c|c|c|}
\hline & $\mathrm{N}^{\circ}$ de pares & $\mathrm{r}$ Spearman & $\mathrm{P}$ & \\
\hline MUI vs MAU & 23 & -0.1846 & 0.3990 & no significativa \\
\hline $\mathrm{SM+MI}$ (max)* vs MAU & 23 & 0.2770 & 0.2008 & no significativa \\
\hline SM+MI (prom)** vs MAU & 23 & 0.2269 & 0.2979 & no significativa \\
\hline MGUI vs MAU & 21 & -0.09507 & 0.6819 & no significativa \\
\hline
\end{tabular}

estimaciones para segmentos proximales y distales y no para huesos enteros (ver Borrero, 1990; Lyman, 1992, 1994) ${ }^{2}$. Para estas adaptaciones se utilizó el valor más alto consignado entre el distal y el proximal de un mismo elemento o el promedio entre los valores de ambos extremos. Los resultados de las correlaciones se consignan en la Tabla 5.

En ningún caso estas correlaciones arrojan resultados significativos, pero es destacable que en todos ellos los valores tienden a elevar el valor de $r$, incluso mostrando una leve tendencia positiva. Respecto de la clasificación propuesta por Lyman, esta combinación de resultados ubica unívocamente este caso en la Clase 4, excluyendo la combinación que lo encuadra en la Clase 1. Por ende, se atenúa el problema de la equifinalidad y la balanza se inclina hacia la destrucción diferencial de elementos con bajo potencial de supervivencia como uno de los factores determinantes en la conformación del conjunto.

De manera independiente a los bajos niveles de correlación con los índices de rendimiento, las evidencias que sugieren que la integridad del conjunto fue afectada por la acción de procesos atricionales se mantiene. En otras palabras, la evidencia indica que aquellos sectores anatómicos de baja densidad $y$ alto retorno económico, fundamentalmente vértebras y costillas, están presentes en bajas proporciones. De esta observación se deriva que si la destrucción diferencial ha afectado sensiblemente el conjunto, las posibilidades de detectar pautas de selección que prioricen el transporte de segmentos anatómicos de alto rendimiento son lejanas.

A fin de sortear estas situaciones Marean y Cleghorn propusieron la aplicación del Modelo de

2 En las publicaciones consultadas solo se dispone de índices estandarizados para extremos proximales $y$ distales, sin consignar estimaciones para huesos completos o diáfisis. Tampoco se presentan los pesos
Alto y Bajo Potencial de Supervivencia (Marean \& Cleghorn, 2003; Cleghorn \& Marean, 2004, para una aplicación en el ámbito patagónico ver Otaola, 2010). Este modelo se basa sobre dos puntos básicos: el primero es la existencia de un "umbral" en la escala de densidad ósea a partir del cual las expectativas de sobrevivencia de un elemento son mucho mayores. Por encima de este umbral quedan incluidas las diáfisis de huesos largos, cráneos y mandíbulas (debido a la presencia de dientes y cóndilos) y otros elementos densos como los astrágalos y calcáneos. Por debajo de este umbral se ubican fundamentalmente las vértebras y las costillas, además de otros elementos de baja densidad. El segundo punto está determinado por la distribución del hueso esponjoso en el esqueleto o en los diferentes elementos y el carácter de "atractivo" y "accesible" para los carnivoros que presentan estas porciones anatómicas. En consecuencia, la conjugación de estas dos condiciones -baja densidad y abundancia de tejido esponjoso- en diversas partes del esqueleto resulta en sectores con menores expectativas de supervivencia que otros donde estas condiciones no están presentes. Esto permite a los autores determinar la existencia de dos grandes sets de huesos, denominados conjuntos de Bajo y Alto Potencial de Supervivencia. Proponen incluir en las correlaciones con los índices de rendimiento solo el conjunto de Alto Potencial de Supervivencia, eliminando de esta manera el sesgo producido por la baja frecuencia de elementos incluidos en el conjunto con menor expectativa de preservación (Marean \& Cleghorn, 2003; Cleghorn \& Marean, 2004).

en bruto por elemento, que permitirían adaptar las estimaciones a los requerimientos de esta instancia analítica mediante los procedimientos discutidos por Metcalfe y Jones (1988). 
Tabla 6. Resultados de correlación $\mathrm{r}$ de Spearman con índices de rendimiento económico y el MAU calculado a partir del set de alta potencialidad de supervivencia. (max.)* y (prom.)** ídem tabla 5.

\begin{tabular}{|c|c|c|c|c|}
\hline & $\mathrm{N}^{\circ}$ de pares & $\mathrm{r}$ Spearman & $\mathrm{P}$ & \\
\hline MUI vs MAU & 11 & 0.2258 & 0.5034 & no significativa \\
\hline SM+MI (max.)* vs MAU & 11 & 0.2694 & 0.4181 & no significativa \\
\hline SM+MI (prom.)** vs MAU & 11 & 0.1644 & 0.6337 & no significativa \\
\hline MGUI vs MAU & 11 & 0.3684 & 0.2608 & no significativa \\
\hline
\end{tabular}

Dado que los perfiles de representación anatómica de guanaco descriptos se ajustan muy bien a aquellas situaciones para las cuales fue diseñado este modelo, se consideró apropiada su aplicación en el análisis de este caso. Para esta instancia analítica se excluyeron de las correlaciones aquellos elementos que componen el conjunto de Bajo Potencial de Supervivencia, considerando solo las estimaciones para huesos largos completos (húmero, radiocúbito, metacarpo, fémur, tibia, metatarso), además de cráneo, mandíbula, astrágalo, calcáneo y primera falange. Los resultados pueden apreciarse en la Fig. 4 y Tabla 6.

Incluso con la aplicación de este modelo no se detectan altos niveles de correlación entre las variables, aunque es importante enfatizar que la tendencia se invierte claramente respecto de la instancia analítica inicial. En todos los casos los valores de $r$ se elevan y tienden a manifestar leves correlaciones de signo positivo, lo que indica una cierta incidencia en el conjunto de elementos de medio y alto rendimiento. Pese a la ausencia de significación, puede observarse que la distribución

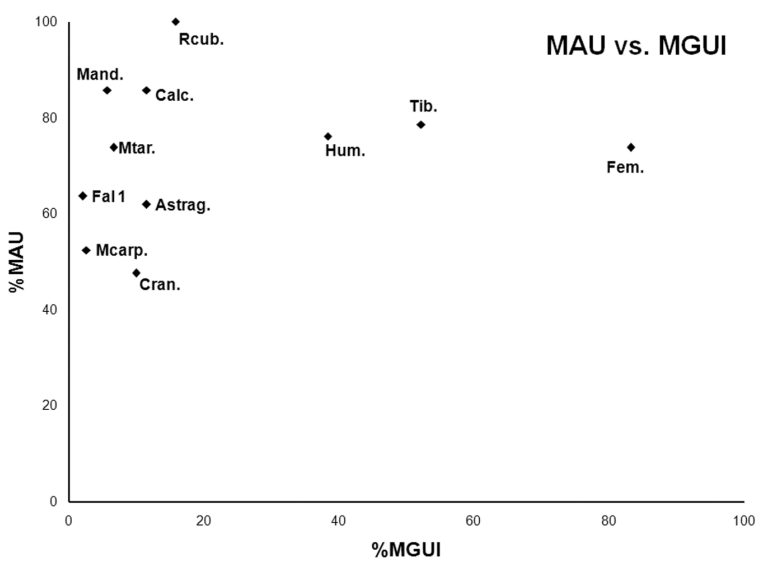

Fig. 4. Gráfico de la correlación entre MAU y MGUI, con la utilización del set de Alto Potencial de Supervivencia. de puntos en los gráficos correspondientes muestra una cierta identidad con las curvas que ilustran el modelo de estrategia global o bulk strategy (Fig. 4), en el cual la selección de partes a transportar recae en porciones de medio y alto retorno económico (Binford, 1978). Si bien aún con los incrementos resultantes de la aplicación del modelo de Bajo y Alto Potencial de Supervivencia, en sentido estricto, este conjunto se mantiene dentro de la clase 4 de Lyman, para la cual la incidencia de los procesos atricionales es gravitante, recordemos que los análisis iniciales tendían a concordar con la noción del descarte en el sitio de elementos de bajo rendimiento. No obstante, como se discutirá más adelante esta ausencia de patrones de selección "limpios" y bien determinables podría estar relacionada con la baja resolución que implica el análisis de un conjunto de la magnitud de la capa $\mathrm{D}$ de Túnel I. Por último, es necesario recordar que el grado de significación de las correlaciones aumentará en la medida que las dos series de variables tiendan a ajustarse de manera lineal, situación característica de la curva que ilustra el modelo Unbiased o su inversa. Pero los casos que se asemejen a las curvas que modelizan las estrategias denominadas Bulk, Gourmet o sus manifestaciones inversas, posiblemente no se ajusten a correlaciones estadísticas con altos niveles de significación y en estos casos la visualización de las distribuciones de puntos podrían ser más informativas que los resultados de las correlaciones por sí solos.

\section{Faenamiento y carnívoros: cortes, golpes y mordidas}

En esta sección se presentan los resultados de los análisis de modificaciones sobre las superficies óseas. Se busca caracterizar los patrones de faenamiento y explorar estos datos en función de 


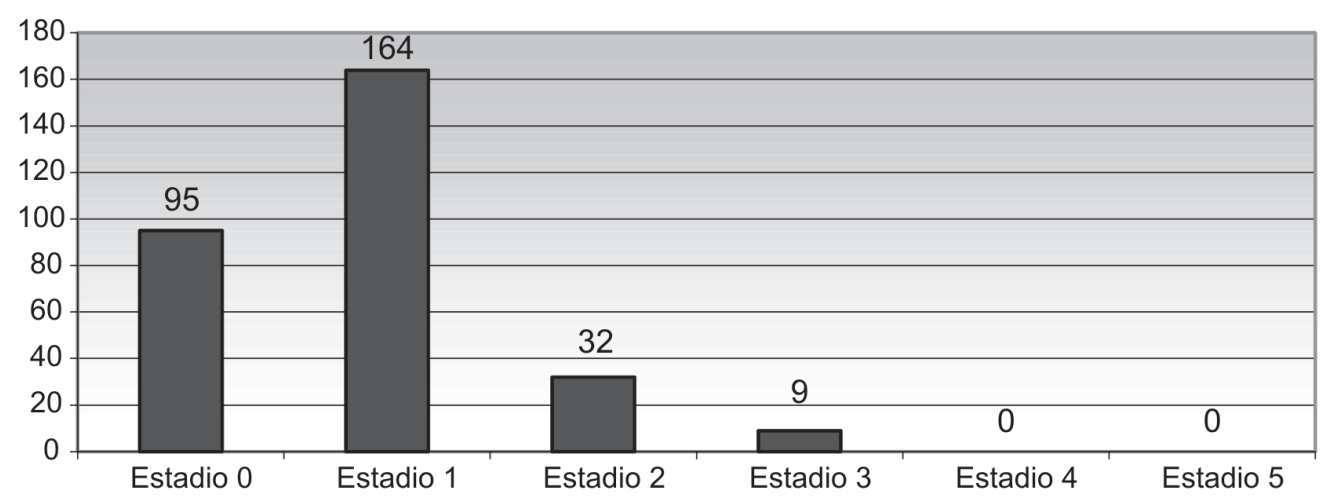

Fig. 5. Perfil de meteorización.

comprender en mayor profundidad el rol que han tenido los diversos agentes en la conformación del conjunto. Nos focalizaremos en la cuantificación por segmentos anatómicos de huellas de corte e impacto y marcas de carnívoros, dejando para otra ocasión una presentación y discusión más profunda acerca de los tipos y asignaciones funcionales de estas modificaciones. La determinación de las modificaciones fue efectuada a ojo desnudo y con bajos aumentos, utilizando una lupa binocular de entre 6X y 50X.

En general la preservación de las superficies óseas de los especímenes recuperados en los depósitos conchíferos del Beagle es muy buena, en parte debidoalPh neutro de estos depósitos(Orquera \& Piana, 2000 y observaciones personales). El caso del Segundo Componente de Túnel I no es la excepción. El perfil de meteorización fue realizado sobre una muestra de 300 especímenes, incluyendo elementos completos y fragmentos de todas las regiones anatómicas del esqueleto. Las mayores frecuencias se registraron en los estadios 0 y 1 (86,3\%) de Behrensmeyer (1978), no habiéndose consignado casos en los estadios más avanzados de meteorización (Fig. 5). Este perfil de meteorización tiene dos implicancias: primero el estado de preservación es suficientemente bueno como para que la identificación de modificaciones sobre las superficies óseas brinde resultados confiables y representativos; segundo, indica que debemos descartar la destrucción in situ por exposición prolongada como causa de la pérdida de elementos.

La baja frecuencia de elementos con menor potencial de preservación demostrada anteriormente debería entonces estar relacionada con la acción de otros agentes naturales o con la intensidad del faenamiento humano. En este sentido se exploraron las evidencias del procesamiento humano y de la acción de carnívoros en búsqueda de clarificar las causas. Ya fue mencionado anteriormente que los índices que se desprenden de la relación NISP/MNE indican la existencia de un importante nivel de fragmentación en el conjunto.

Todas las regiones anatómicas muestran evidencias de faenamiento de diversa índole -corte, descarne, impacto, machacado-, las frecuencias de especímenes con huellas son variables por elemento y porción. La correlación entre los valores de NISP y el porcentaje de marcas de corte por elemento (Lyman, 1994, p. 310-311) arroja resultados no significativos $\left(r_{s}=-0,1443 ; \mathrm{P}=0,3500\right)$, por lo tanto no hay razón para sospechar que la frecuencia de marcas en un determinado segmento anatómico dependa de su representación en el conjunto. El detalle de las cantidades de especímenes y las frecuencias de marcas de corte e impactos puede observarse en la Tabla 7 y la Fig. 6. Todas las regiones anatómicas han sido explotadas en forma intensiva, los porcentuales de corte más frecuentes se ubican entre un 25 y $50 \%$ del NISP y globalmente el valor registrado para el conjunto total es de $31,1 \%$. La variabilidad morfológica, ubicación anatómica y orientación de marcas de corte e impacto registradas denotan que los huesos han atravesado diferentes estadios de faenamiento antes de su descarte, lo cual es coherente con la interpretación del sitio como campamento doméstico o residencial. En comparación con las asignaciones funcionales de tipos de marcas, basadas sobre trabajos etnoarqueológicos y experimentales (Binford, 1981; Lyman, 1987), 
se registraron marcas relacionadas a acciones de extracción de cueros, desarticulación y descarne. A su vez no hay casos de huesos largos o cilindros diafisarios completos y la frecuencia de huellas de impacto sobre los fragmentos de diáfisis es alta (Tabla 7).

En cuanto a la distribución anatómica de las huellas de corte, estas tienden a presentar mayores frecuencias en los elementos de los miembros, especialmente en el posterior. Aunque también es este segmento anatómico el que muestra mayor variabilidad interna con valores que fluctúan entre el $62,6 \%$ en la diáfisis del fémur y $10,1 \%$ en la sección distal del mismo hueso; uno de los porcentuales más bajos registrados en todo el esqueleto. Esta marcada diferencia puede estar vinculada con la extracción de masas musculares grandes adjuntas a la parte medial y proximal del fémur y para el caso de la porción distal del mismo elemento, la baja frecuencia de marcas de corte podría relacionarse con la relativa facilidad de desarticulación que presenta la articulación fémur-tibia. También el miembro anterior en conjunto muestra mayores frecuencias que el esqueleto axial, aunque en algunos segmentos de la columna y la cabeza se registraron altas proporciones de elementos con huellas (cuello y mandíbulas). Si incluimos en estas consideraciones las marcas de impacto, claramente más representadas en las diáfisis de huesos largos, en relación con la fractura para el consumo de médula, el patrón que indica una mayor intensidad

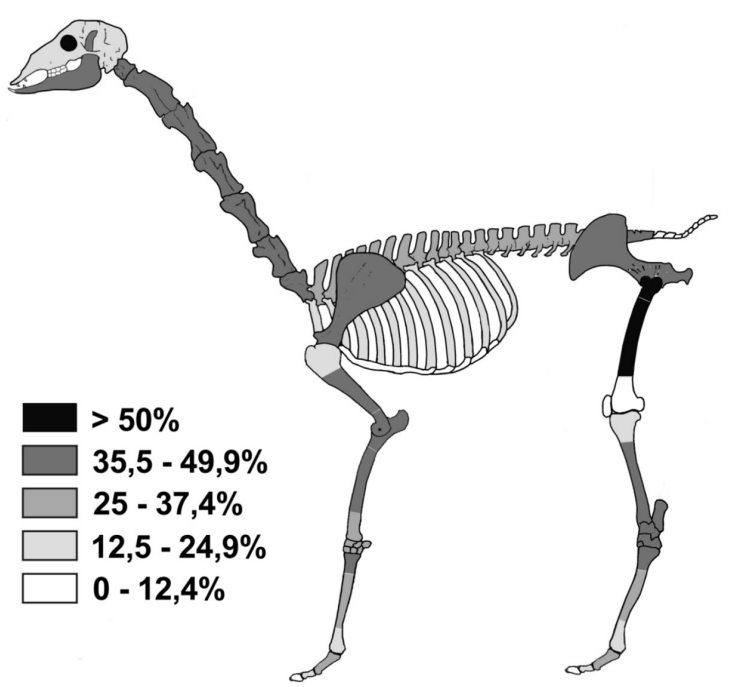

de aprovechamiento de los miembros se acentúa notablemente.

En relación con la diversidad de huellas de corte $y$, solo a manera informativa, podemos mencionar que la extracción de cueros está atestiguada por la presencia de marcas transversales y profundas sobre segmentos distales de metapodios (Fig. 7A). Muchas marcas asociadas directamente con zonas articulares se relacionan con acciones de desarticulación (Fig. 7B). La extracción de músculo también fue registrada en diversos sectores, entre otros: extracción de lengua (cortes longitudinales y machacado en caras internas de mandíbula) (Fig. 7C), remoción de musculatura apendicular (cortes longitudinales largos sobre diáfisis de fémures, hojas de escápulas, etc.) (Fig. 7D y E), extracción de músculos dorsales (cortes longitudinales sobre espinas dorsales de vértebras toráxicas y lumbares). Los fragmentos de diáfisis de huesos largos presentan altas frecuencias de muescas de impactos y fracturas helicoidales que indican un intensivo aprovechamiento de médula. En sintesis: todas las partes de la anatomía de las presas parecen haber sido aprovechadas en forma intensiva; la adición y superposición de indicadores funcionales de distintas etapas de la secuencia obtención-faenamiento-consumo-descarte indican la posición terminal de este conjunto en la cadena de explotación.

Por su parte, también los carnivoros parecen haber actuado de manera intensa, aunque

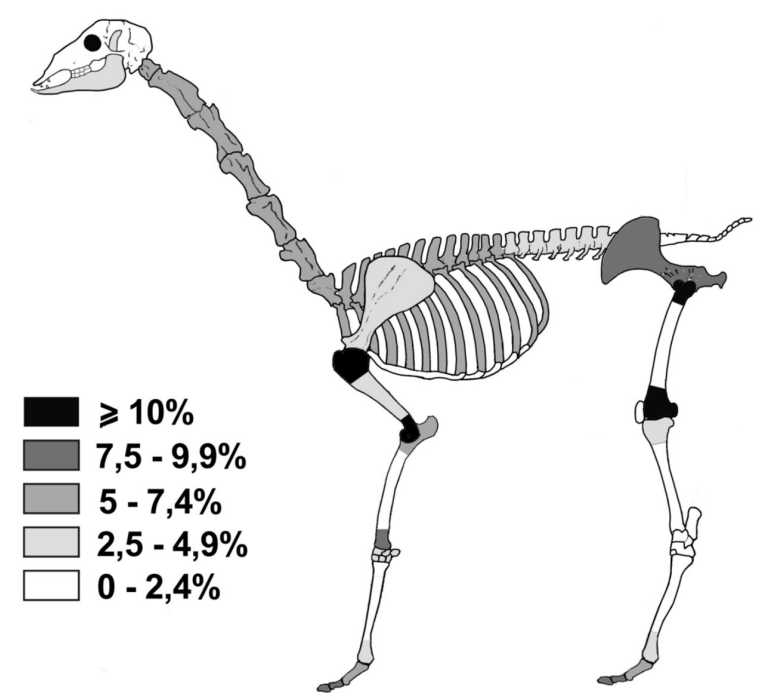

Fig. 6. Izquierda: sintesis de los porcentuales de marcas de corte. Derecha: de carnívoro. 
Tabla 7. Porcentaje de especímenes con marcas de corte e impacto y acción de carnívoros.

\begin{tabular}{|c|c|c|c|c|c|}
\hline \multicolumn{2}{|c|}{ Unidad Anatómica } & NISP & NISP Corte & NISP Impacto & NISP Carniv. \\
\hline \multirow{3}{*}{ Cabeza } & Cráneo & 171 & 28 (16.37\%) & 0 & $4(2.33 \%)$ \\
\hline & Mandíbula & 130 & 47 (36.1\%) & $18(13.9 \%)$ & $4(3.07 \%)$ \\
\hline & Sub total & 301 & 75 (24.91\%) & $18(5.98 \%)$ & $8(2.65 \%)$ \\
\hline \multirow{9}{*}{ Axial } & Vert. Atlas & 17 & 7 (41\%) & 0 & $1(5.88 \%)$ \\
\hline & Vert. Axis & 18 & 7 (38.88\%) & $5(27.77 \%)$ & $1(5.55 \%)$ \\
\hline & Vert. Cervical & 193 & 79 (40.93\%) & 0 & $13(6.73 \%)$ \\
\hline & Vert. Torácica & 150 & 51 (34.00\%) & $18(12 \%)$ & $9(6 \%)$ \\
\hline & Vert. Lumbar & 237 & 69 (29.11\%) & 35 (14.76\%) & 7 (2.95\%) \\
\hline & Sacro & 21 & $8(38 \%)$ & 3 (14.28\%) & 0 \\
\hline & Pelvis & 143 & 56 (39.16\%) & $38(26.57 \%)$ & $11(7.69 \%)$ \\
\hline & Costilla & 451 & $84(18.62 \%)$ & 0 & 23 (5.09\%) \\
\hline & Sub total & 1230 & 361 (29.34\%) & 99 (8.04\%) & $65(5.28 \%)$ \\
\hline \multirow{9}{*}{ Miembro Anterior } & Escápula & 92 & 38 (41.30\%) & 10 (10.86\%) & $3(3.26 \%)$ \\
\hline & Húmero px & 36 & $8(22.2 \%)$ & 0 & 5 (13.88\%) \\
\hline & Húmero df & 107 & 45 (42.05\%) & 39 (36.44\%) & $4(3.73 \%)$ \\
\hline & Húmero ds & 50 & $22(44 \%)$ & 5 (10\%) & $5(10 \%)$ \\
\hline & Radiocúb. px & 82 & 33 (40.24\%) & $21(52.60 \%)$ & $6(7.31 \%)$ \\
\hline & Radiocúb. df & 148 & 56 (37.83\%) & 71 (47.97\%) & $2(1.35 \%)$ \\
\hline & Radiocúb. ds & 81 & $24(29.62 \%)$ & $4(4.93 \%)$ & 7 (8.64\%) \\
\hline & Metacarpo px & 56 & $26(46.42 \%)$ & 27 (48.21\%) & 0 \\
\hline & Sub total & 652 & 252 (38.65\%) & 177 (27.14\%) & 32 (4.90\%) \\
\hline \multirow{8}{*}{ Miembro Posterior } & Fémur px & 43 & $24(55.8 \%)$ & $4(9.3 \%)$ & $5(11.62 \%)$ \\
\hline & Fémur df & 107 & 67 (62.61\%) & $60(56.07 \%)$ & 0 \\
\hline & Fémur ds & 48 & 5 (10.41\%) & $1(2.08 \%)$ & $6(12.50 \%)$ \\
\hline & Tibia px & 66 & 13 (19.69\%) & $1(1.51 \%)$ & 7 (10.6\%) \\
\hline & Tibia df & 107 & $50(46.72 \%)$ & 56 (52.3\%) & $4(3.73 \%)$ \\
\hline & Tibia ds & 38 & 15 (39.47\%) & $4(10.52 \%)$ & 0 \\
\hline & Metatarso px & 58 & $24(41.37 \%)$ & 32 (55.17\%) & 0 \\
\hline & Sub total & 467 & 198 (42.39\%) & 158 (33.83\%) & $22(4.71 \%)$ \\
\hline \multirow{6}{*}{$\begin{array}{c}\text { Autopodios } \\
\text { indiferenciados }\end{array}$} & Metapodio df & 192 & $54(28.12 \%)$ & 97 (50.52\%) & $4(2.08 \%)$ \\
\hline & Metapodio ds & 125 & 23 (18.4\%) & $14(11.2 \%)$ & $6(4.80 \%)$ \\
\hline & $1^{\circ}$ Falange & 279 & 76 (27.24\%) & 54 (19.35\%) & 20 (7.16\%) \\
\hline & $2^{\circ}$ Falange & 146 & 27 (18.49\%) & $19(13.01 \%)$ & 13 (8.90\%) \\
\hline & $3^{\circ}$ Falange & 51 & $5(9.80 \%)$ & 5 (9.80\%) & $5(9.80 \%)$ \\
\hline & Sub total & 793 & 185 (23.32\%) & $189(23.83 \%)$ & $48(6.05 \%)$ \\
\hline \multicolumn{2}{|c|}{ TOTAL } & 3443 & 1071 (31.10\%) & 641 (18.61\%) & 175 (5.08\%) \\
\hline
\end{tabular}

dispar y selectiva; se destaca la recurrencia de sus marcas en los extremos articulares de algunos huesos largos y en regiones del esqueleto axial. Particularmente sobresalen las altas frecuencias determinadas en extremos proximales de húmeros y fémures, así como en la porción proximal de la tibia. El cálculo global indica que poco más del $5 \%$ del conjunto está afectado por este tipo de marcas, valor que puede considerarse alto en el contexto de la información disponible (ver Muñoz, 2009). 


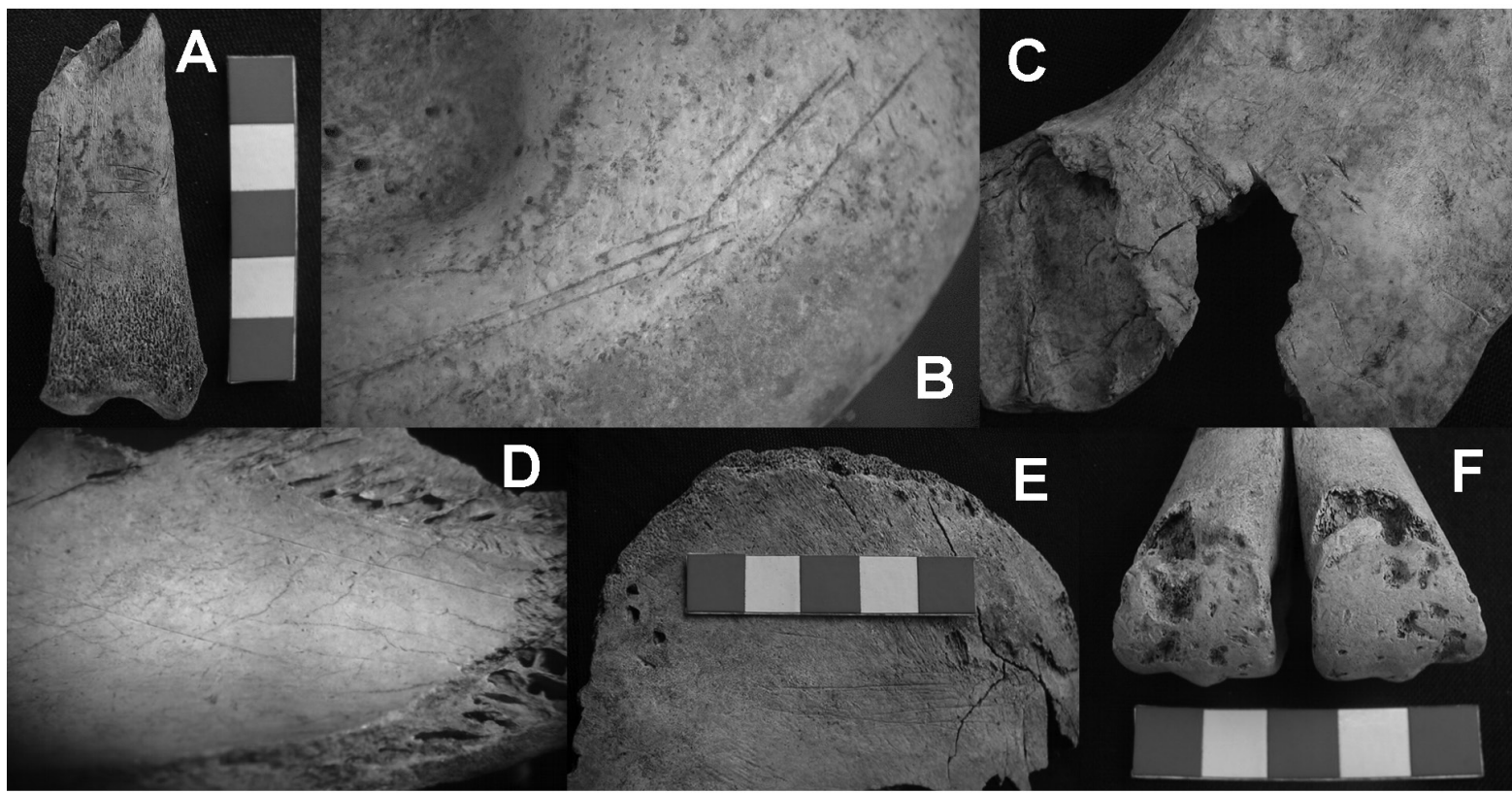

Fig. 7. A: huellas de corte transversales en cara anterior de metapodio distal. B: huellas de corte sobre cara lateral de cóndilo distal de húmero, relacionadas con la desarticulación de húmero y radiocúbito. C: huellas de corte y machacado (chopmarks) en caras internas de mandíbula. D: huellas de corte longitudinales largos sobre hoja de escápula. E: huellas de corte sobre superficie externa de hoja del íleon y marcas de carnívoro (perforaciones) y borde crenulado sobre cresta iliaca. F: marcas de carnivoros sobre trócleas de metapodio.

Recordemos que en Tierra del Fuego no existieron grandes carnivoros, el único presente con seguridad durante el Holoceno ha sido el zorro colorado (Lycalopex culpaeus), de unos $11 \mathrm{~kg}$ y 55 $\mathrm{cm}$ de alto y potencialmente Dusicyon avus, zorro extinto de mayor tamaño -entre 12 y $15 \mathrm{~kg}$ - para el cual no se han detectado evidencias provenientes del sur de Tierra del Fuego, pero sí se han obtenido edades de hasta principios del Holoceno tardío en el norte de la Isla Grande (Prevosti et al. 2011). Pese a que varios autores han discutido el rol de los zorros como factor de distorsión del registro desde

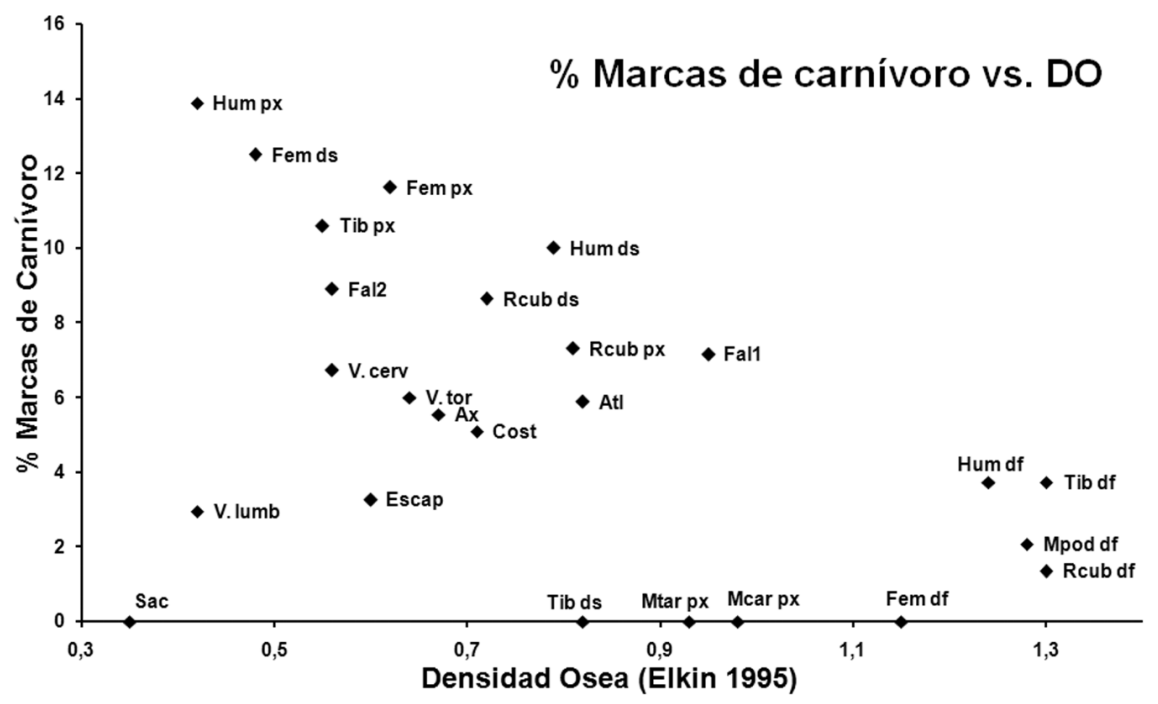

Fig. 8. Gráfico de la correlación entre el porcentual de marcas de carnívoros detectado y la DMO. 
una perspectiva tafonómica para casos fueguinos (ver Borrero, 2007a; Estévez Escalera \& Mameli, 2000; Muñoz, 2009); los estudios sobre su acción en conjuntos arqueológicos de la región del Beagle aún no han sido profundizados.

La frecuencia de marcas de carnívoros fue correlacionada con la DMO para explorar la posibilidad que la pérdida de elementos estuviera relacionada con la acción de estos animales. La significación de la correlación entre ambas variables $\left(r_{s}=-0,4605, p=0,0205\right)$ indica un aumento en la frecuencia en aquellas porciones de menor densidad (Fig. 7F y 8).

Estos resultados subrayan la pertinencia de la aplicación del modelo de Bajo y Alto Potencial de Supervivencia utilizado, ya que las frecuencias de mordidas son coherentes con el carácter atractivo y accesible que presentan los segmentos anatómicos de baja densidad para los carnívoros. Más aún en el caso fueguino, en donde la potencia de las mordidas de los zorros no les permite fracturar huesos de alta resistencia (Muñoz, 2009). Sin embargo, si bien estos resultados son sugestivos no puede pasar desapercibido que el potencial de detección de marcas de carroñeo en aquellos segmentos menos densos es mayor que el esperable en segmentos más densos como las diáfisis. Entonces este patrón podría estar "mapeando" la susceptibilidad estructural de los huesos a ser marcados por mordidas antes que una pauta de comportamiento de los carnívoros.

\section{DISCUSIÓN Y CONCLUSIONES}

En la arqueología de la región del canal Beagle la investigación zooarqueológica y tafonómica están en pleno desarrollo. Próximamente se podrá contar con más trabajos específicos de clases de recursos y análisis de conjuntos que sin dudas aportarán datos interesantes a la interpretación de la variabilidad y los patrones regionales de explotación de vertebrados. Si bien tradicionalmente ha existido una preocupación por la caracterización de las pautas de subsistencia y los modos de adaptación canoera (Piana, 1984; Orquera \& Piana, 1999), por el momento son escasos los análisis zooarqueológicos y tafonómicos de detalle como para plantear generalizaciones o realizar comparaciones con los resultados aquí presentados. En este sentido, este análisis posee aún pocos parámetros de comparación sobre los modos de explotación de los guanacos, como tampoco acerca de tendencias tafonómicas de conjuntos similares que permitan avanzar en discusiones de alcance regional. No obstante a continuación se discute una serie aspectos que considero relevantes a diferentes escalas. Por un lado se destacan aquellos relacionados con los procedimientos analíticos empleados y su potencial aplicabilidad a otros casos de la región. Por otra parte se discuten las implicancias de los resultados aquí presentados tanto a la escala del Segundo Componente de Túnel I, como algunas similitudes y diferencias detectadas con las informaciones disponibles para otros conjuntos de guanaco en el sur de Tierra del Fuego.

Aspectos analíticos de la aplicación de modelos tafonómicos

A lo largo del desarrollo de este trabajo la puesta en práctica de diversos modelos tafonómicos permite arribar a una serie de reflexiones sobre su pertinencia en este caso y su potencial aplicabilidad a otros conjuntos de la región. Entre ellas, las que considero más relevantes se relacionan con los procedimientos de cuantificación y sus implicancias en la interpretación de pautas de transporte o conservación diferencial. La exploración de diferentes alternativas de cuantificación y su comparación con los modelos de rendimiento, principalmente en aquellos aspectos relacionados con la representatividad de las diáfisis, fue una de las preocupaciones que guiaron este trabajo. Se evalúa como apropiada la aplicabilidad del modelo de Alto Potencial de Supervivencia en las correlaciones con los índices económicos (Cleghorn \& Marean, 2004; Otaola, 2010).

En numerosos conjuntos de guanacos procedentes de Fuego-Patagonia ha sido documentada la existencia de perfiles anatómicos en los cuales dominan los elementos de los miembros y la cabeza (Mengoni Goñalons, 1999; Muñoz, 2001; Santiago, 2013; entre otros), situación que comienza a manifestarse también en los contextos procedentes de la región del canal Beagle. Comúnmente este patrón ha sido explicado en términos de decisiones humanas y son escasos 
los conjuntos en que se ha incluido a la destrucción diferencial de elementos como parte de estas interpretaciones. Generalmente la baja incidencia de evidencia de agentes perturbadores: marcas de carnívoros, roedores, pisoteo, meteorización, etc., en conjunción con la ausencia de correlaciones positivas con la densidad mineral ósea, han sido las razones que sustentaron la exclusión de la destrucción de elementos de las ecuaciones tafonómicas. Sin embargo, en muchos de estos casos tampoco se han expresado de manera univoca las tendencias que permitieran encuadrar estos patrones dentro de los distintos modelos de transporte selectivo. En este contexto parece prometedora la idea de profundizar la exploración desde la óptica de un modelo que considera la expectativa de supervivencia, de un patrón en el cual "por definición" se excluyen o se presentan en bajas proporciones las regiones de mayor rendimiento. En otras palabras: sería improbable detectar patrones de selección claros en función del rendimiento económico en casos en los cuales las regiones de alto retorno no están presentes. En consecuencia, se espera que aún reduciendo la representatividad anatómica del análisis, el uso de un subconjunto no tan condicionado por la supervivencia diferencial podría arrojar resultados más ajustados.

En el caso del Segundo Componente de Túnel I la hipótesis de funcionalidad más parsimoniosa y avalada por la evidencia contextual es que sus ocupaciones pueden caracterizarse como las propias de un campamento base o de actividades múltiples. En consecuencia las expectativas indicaban que -trozamiento, selección y transporte mediante- fuera hacia Túnel donde se llevaran las partes seleccionadas de los guanacos cazados y no que fuera en este sitio donde se descartaran partes de escaso rendimiento económico. Sin embargo, las primeras instancias analíticas basadas sobre la cuantificación de los extremos de los huesos largos indicaron una tendencia negativa -si bien con nulo o escaso nivel de significación- entre los valores de representación anatómica y los de utilidad económica (Tabla 4). Complementando esta situación, las frecuencias de elementos se ajustan muy bien a la escala de DMO, lo que señala la destrucción de partes de baja densidad, ubicando al conjunto en el ámbito de la equifinalidad
(Lyman, 1992, 1994). Resultados similares han sido reportados para arqueofaunas de diversas partes del mundo, los que llevaron a Lyman (1985, 1992) y a Grayson (1989) a proponer que tales combinaciones de curvas reflejarían una situación de destrucción mediatizada por la densidad mineral antes que decisiones humanas respecto de qué partes transportar (ver Marean \& Frey, 1997).

Ahora bien, si utilizamos modelos tafonómicos desarrollados originalmente para sortear estas aparentes incongruencias, específicamente el modelo de Bajo y Alto Potencial de Supervivencia, las tendencias previas se modifican sustancialmente. La exclusión de elementos de baja densidad ósea y alta proporción de tejido trabecular de las correlaciones con los indices de rendimiento muestra un panorama muy distinto del anterior, volcando la tendencia hacia una mejor representación de partes de medio y buen rendimiento económico. Como fue destacado, tales correlaciones no manifiestan altos grados de significancia, por lo tanto los patrones emergentes no serían explicables apelando a decisiones estrictamente económicas. Aunque, como también fue considerado más arriba, la ausencia de correlaciones significativas no necesariamente invalidan la existencia de selecciones de índole económica.

En relación con esta ausencia de un patrón nítido en la selección de segmentos de buen retorno alimenticio, aún resta indagar en situaciones de mayor resolución dentro del Segundo Componente. En este sentido es necesario tener en cuenta que determinadas pautas de selección y transporte pueden verse oscurecidas por la magnitud y amplitud cronológica del conjunto y que en caso de analizar subconjuntos más reducidos, estos podrían manifestar tendencias donde la evaluación de las decisiones humanas fuese más "visible". Recordemos que la capa D está compuesta por una larga sucesión de ocupaciones que abarcan aproximadamente 2.000 años. Por lo tanto sería muy poco probable que los distintos eventos de cacería se hubiesen dado en contextos situacionales siquiera similares. Por el contrario, es esperable que a lo largo de semejante lapso las presas hubieran sido capturadas, procesadas y transportadas en una gran variabilidad de situaciones: distancia al campamento, cantidades de personas para 
transportar o para alimentar, número de animales cazados, disponibilidad de otras presas, entre otras variables.

En algunos casos, aunque no mayoritaria, la presencia de elementos de bajo rendimiento podría ser interpretada en situaciones de cercanía entre Túnel y los sitios de matanza, lo que implicaría bajos costos de transporte, tendiendo a la implementación de estrategias de máxima (Emerson, 1993) o del tipo Bulk strategy (Binford, 1981). Explicaciones de esta índole han sido postuladas para el caso de contextos recuperados en Estancia Moat, donde es esperable una disponibilidad mayor y más inmediata de estas presas (Alumnni \& Zangrando, 2012). Sin embargo, esta no sería la situación dominante para el área central del canal Beagle, donde la oferta de guanacos sería sensiblemente menor. Pienso que un contexto de las características de la Capa D de Túnel I presenta una superposición de eventos tal que su análisis de conjunto genera invariablemente un panorama difuso. Conjuntos más chicos implican mejor resolución, aunque menor representatividad. Una vía analítica que se está explorando es el estudio de estos patrones discriminando en las ocho Fases de Formación de la Capa D (ver Orquera \& Piana, 1992 y Zangrando, 2009). Cabe destacar que algunos resultados preliminares sugieren que discriminando entre estos subconjuntos se vislumbra cierta variabilidad en los perfiles de abundancia anatómica.

Independientemente de la detección o no de patrones de selección relacionados con situaciones de transporte selectivo, no debemos perder de vista que las evidencias indican fuertemente que este conjunto ha sido mediado por la destrucción diferencial. A la menor representación de elementos del esqueleto axial, debemos sumar las frecuencias de marcas de carnívoros, sobre todo concentradas en sectores de baja densidad ósea, por lo cual no podemos desestimarlos como agentes activos en la formación de este registro. En el contexto del cuerpo de evidencia disponible me inclino a interpretar este contexto como el resultado de una situación terminal en la cadena de explotación, por lo tanto habiendo atravesado todos los estadios posibles de desorganización y destrucción implicados en un procesamiento intensivo, a lo que se le añade una fuerte acción de los zorros postdescarte, y posiblemente luego del abandono del sitio por los humanos. Estos carroñeros habrían accedido a los huesos ya totalmente desarticulados en unidades menores, muy fragmentados, sin carne y sin médula, aunque aun con abundante grasa y nutrientes contenidos en su tejido trabecular. Sin dudas, las localidades con basurales del tipo de los concheros recurrentemente utilizados deben haber concentrado una alta densidad de desechos muy atractivos para los zorros, quienes no perderían oportunidades de carroñar sobre estos puntos con una oferta concentrada de alimento. Además de la destrucción a causa de sus mordidas, estas situaciones debieron implicar la dispersión y perdida de numerosos elementos que los zorros seguramente moverían fuera de su lugar de encuentro.

\section{El aprovechamiento de guanacos en Túnel (6.400-4.500 AP)}

La amplitud cronológica del Segundo Componente (ca. 6.400-4.500 AP), la distribución regular de los restos en el depósito y la baja proporción en comparación con los pinnípedos indican que los guanacos representaron un recurso complementario y ocasional para los ocupantes de Túnel I. Las edades de muerte representadas, el NMI y los patrones de selección y faenamiento sugieren que la explotación de este recurso generalmente se realizó por medio de una estrategia de cacerías oportunistas, en las cuales se obtendrían una o unas pocas presas al mismo tiempo y que estas eran explotadas en forma intensiva $e$ integral (cueros, carne, médula y huesos). Aunque probablemente las situaciones en las cuales se han obtenido, procesado y transportado los guanacos cazados hayan sido muy variables.

Esta escala reducida de explotación pero a su vez exhaustiva de las reses de guanacos tiene implicancias en las decisiones de transporte diferentes que las esperadas en situaciones de matanzas de muchos individuos. En este sentido no sería esperable en Túnel y de hecho no se detectan, patrones de selección muy excluyentes. Como fue mencionado, de este análisis no se pueden derivar patrones claros sobre selección o transporte diferencial solamente en función del rendimiento alimenticio. Sin embargo, las altas 
frecuencias de los miembros, calculada a partir de las porciones diafisarias permiten defender que habría existido cierta preferencia de estos sobre el esqueleto axial. Por el momento y en contra de esta argumentación, tampoco es posible afirmar que los segmentos axiales no hayan sido transportados. La primera razón es la posibilidad que los procesos destructivos -tanto culturales como naturaleshayan eliminado buena parte de estos elementos, además de considerar que tampoco es posible argumentar su total ausencia ya que muchas de estas porciones anatómicas, si bien minoritarias frente a los miembros, alcanzan valores de hasta el 50\% del MAU. Como se dijo, esta ausencia de patrones claros puede estar relacionada con la amplitud del conjunto o con condiciones situacionales diversas a las que se habrían enfrentado los cazadores. En algunos casos puntuales, otros factores, además del rendimiento en carne y grasa, pueden haber influenciado las decisiones humanas en cuanto a qué elementos transportar, conservar o descartar. Uno a tener en cuenta son los requerimientos tecnológicos y la potencial utilización de algunas de las diáfisis una vez agotadas como fuente de alimento.

Todas las partes anatómicas fueron intensamente explotadas, tal como queda evidenciado por las frecuencias y posiciones topográficas de las marcas. También la extracción de médula ha sido recurrente en función de las frecuencias de huellas de fractura de los huesos largos. Será de utilidad contar con datos comparables de conjuntos con composición taxonómica similar y de otros en los cuales el guanaco haya sido explotado más asiduamente; esto nos permitirá explorar la significación de los patrones resultantes de este análisis en la escala inter-sitio. Por el momento, partiendo del caso aquí expuesto, resulta evidente que el transporte y la intensidad de explotación han implicado un alto grado de desorganización de las reses cazadas e influyeron también significativamente en la fragmentación de los elementos.

Por otro lado, la representación de partes del conjunto también podría ser explicada, al menos parcialmente, por la conservación diferencial de elementos de alta densidad como consecuencia de la acción de agentes naturales y culturales. Se sostiene que las condiciones de preservación en los concheros son buenas, como se desprende de datos de meteorización y la buena preservación que presentan las superficies óseas. Sin embargo la acción de los zorros en la conformación del conjunto fue importante, gran parte de la destrucción parece deberse a la acción de sus mordidas, particularmente resultan informativas las altas frecuencias halladas en segmentos anatómicos de baja densidad.

\section{Comparación con otros conjuntos en la escala regional}

Hasta el momento existen cinco sitios emplazados en la costa sur fueguina para los cuales se dispone de cierto nivel de detalle en el análisis de restos de guanaco: los conchales inferiores de Imiwaia (Alunni, 2013), Isla el Salmón (Figuerero Torres \& Mengoni Goñalons, 1986), Shamakush I (Orquera \& Piana, 1996) y Heshkaia 34 y 35 (Alunni, 2013; Alunni \& Zangrando, 2012). De ellos el único con cronología comparable a la capa $\mathrm{D}$ de Túnel I es el correspondiente a los conchales inferiores de Imiwaia I (Capas K, L y M), con fechas que lo ubican entre ca. 5.700 y 6.000 años AP. Los conjuntos restantes son tardíos, con cronologías determinadas entre los 1.800 y 600 años AP. En cuanto a su distribución, estas localidades se emplazan en condiciones microambientales relativamente contrastantes dentro del espectro de variabilidad que presenta la región del Beagle. Estas diferencias se manifiesta tanto en relación con Túnel como entre sí, incluso Heshkaia, la más oriental, podría ser considerada por fuera de la región del Beagle (para una caracterización de la variabilidad ambiental en la región del canal Beagle (ver Orquera \& Piana, 1999 y Zangrando, 2009).

El primer aspecto a destacar es la magnitud de los conjuntos, donde resalta el mayor tamaño del proveniente de Túnel I frente al resto de los casos. En total la sumatoria de los restos provenientes de Imiwaia I, Shamakush I, Hershkaia 35 e Isla el Salmón ( $\mathrm{N}=6.454)$, supera por poco el NISP total de guanacos determinados para la Capa D de Túnel I. Lo cual, en algunos casos implica eventuales sesgos debidos al tamaño de las muestras, pero también situaciones de mayor resolución arqueológica en favor de los conjuntos más pequeños. En cuanto a los intervalos temporales determinados en la 
formación de estos conjuntos, también en todos los casos son significativamente menores a la amplitud del Segundo Componente de Túnel I.

La comparación entre los resultados obtenidos para las capas $\mathrm{K}, \mathrm{L}$ y $\mathrm{M}$ de Imiwaia I (Alunni, 2013), con el conjunto recuperado de la Capa D de Túnel I muestra una serie de semejanzas importantes. Entre estas, posiblemente la más significativa sea la mejor representación del esqueleto apendicular frente al axial, incluso en alguno de los conjuntos de Imiwaia mostrando mayor contraste aun que el caso de Túnel. No obstante también es destacable que al menos para las capas $\mathrm{M}$ y $\mathrm{K}$-las cuales presentan valores de NISP más altos- se verifique una tendencia hacia una mejor representación de los segmentos toráxicos y lumbares de la columna vertebral que en Túnel (Alunni, 2013).

Las correlaciones realizadas con los índices de rendimiento anatómico arrojan resultados similares a los obtenidos inicialmente para el caso de Túnel: correlaciones de signo negativo, aunque no significativas. Lo que sugiere a la autora la ausencia de transporte diferencial relacionado con el rendimiento económico; sin embargo, al igual que en Túnel la mayor incidencia de elementos de los miembros podría relacionarse con una estrategia orientada a facilitar el transporte (Alunni, 2013, p. 112). Por su parte, las pautas de procesamiento evidenciadas por la frecuencia, variabilidad $y$ superposición de marcas interpretables como consecuencia de distintas acciones: desarticulación, descarne, extracción de médula, etc., indican, al igual que en Túnel I, la posición terminal de Imiwaia en la cadena de explotación de los guanacos. No obstante, en términos globales la intensidad del procesamiento evidenciada por los porcentajes de elementos con huellas antrópicas es algo mayor en Túnel I.

La similitud de los perfiles de meteorización entre Imiwaia y Túnel denota un proceso de enterramiento relativamente rápido y homogéneo, lo cual se relaciona con la naturaleza de los depósitos, ambos concheros de gran magnitud.

Sin embargo, también se destacan algunas diferencias marcadas. En primer lugar, los conjuntos de Imiwaia muestran valores de integridad anatómica más bajos que Túnel, lo que podría indicar una mayor selectividad o bien podría relacionarse con cuestiones de resolución y tamaño de la muestra. En segundo lugar, la ausencia de significación en las correlaciones entre la abundancia anatómica y la densidad ósea y la escasa representación de marcas de carnivoros llevan a la autora a descartar la influencia de los procesos atricionales en la conformación de los conjuntos (Alunni, 2013). Por último, la clara tendencia registrada en Túnel hacia la mejor representación de diáfisis verificada en todos los huesos largos no se expresa de forma tan manifiesta en los conjuntos de Imiwaia. Alli varios casos presentan mayores frecuencias de extremos proximales o distales que de las correspondientes diáfisis. Situación que podría vincularse a una menor incidencia de los procesos atricionales, o bien a diferencias en los criterios de determinación y cuantificación.

Shamakush I es otro caso donde el análisis de los restos de guanaco ha sido realizado con cierto grado de detalle (Orquera \& Piana, 1996), aunque no se cuenta con el mismo tipo de información que la disponible para Imiwaia o la aquí presentada para la Capa D de Túnel I. La principal diferencia que se manifiesta en la comparación con Imiwaia y Túnel es que en Shamakush I los guanacos constituyen el principal recurso explotado alcanzando un $64,6 \%$ del NISP (Orquera \& Piana, 1996, p. 234). Por otro lado, los autores informan sobre la presencia en el conjunto de todas las partes anatómicas, aunque la ausencia de cuantificaciones impide comparaciones más detalladas. Tanto para el caso de Túnel como el de Imiwaia también se ha mencionado la presencia de todas las partes anatómicas, pero las cuantificaciones por elemento han mostrado que esta representación es muy variable. Ante la ausencia de tales cuantificaciones y la mención sobre la presencia de todos los segmentos anatómicos, podría asumirse que posiblemente esto indique una menor incidencia de transporte o destrucción diferencial para el caso de Shamakush, aunque por el momento resulta aventurado realizar cualquier afirmación. En Shamakush I no se han llevado a cabo estudios tafonómicos, por lo que no es posible incorporar este conjunto en la discusión sobre estos aspectos.

Los contextos provenientes de Isla el Salmon (Figuerero Torres \& Mengoni Goñalons, 1986) son muy pequeños en comparación con los casos aquí tratados, menos de 50 especímenes 
para cada uno de los sitios excavados (Isla el Salmón 5 y 6), lo que implica posibles sesgos relacionados con el tamaño de la muestra. Cabe destacar la mayor representación de elementos de las patas y la cabeza, lo que lleva al autor a proponer la existencia de una selección de partes, aunque esta no estaría directamente relacionada con el rendimiento económico, ni tampoco con la destrucción diferencial de elementos (Mengoni Goñalons, 1986:65-66).

Las similitudes entre los conjuntos de Hershkaia e Imiwaia ya fueron destacadas en otra ocasión (Alunni, 2013), en general se sostiene que tanto los patrones anatómicos y las evidencias de procesamiento, como las improntas tafonómicas observadas son similares en ambas localidades. Por lo cual en líneas generales se pueden extrapolar las consideraciones realizadas en la comparación entre Túnel e Imiwaia, también a los conjuntos de Hershkaia. En los conjuntos analizados en esta localidad, al igual que en Imiwaia y Túnel se destaca la mejor representación de los miembros, la variabilidad y superposición del tipo de marcas, la práctica generalizada de explotación de médula en huesos largos y la ausencia de patrones de selección en función del rendimiento económico. Por otra parte, al igual que los conjuntos de Imiwaia denotan una baja incidencia de procesos atricionales, lo cual los diferencia del caso de la capa $\mathrm{D}$ de Túnel.

A manera de conclusión general cabe puntualizar que la evidencia disponible sobre la explotación de guanacos proveniente de la región de canal Beagle y del Segundo Componente de Túnel I en particular, denota la existencia de una pauta que habría priorizado la selección de los miembros, lo que sugiere una tendencia a minimizar costos de transporte. En todos los casos las estrategias de obtención habrian sido oportunistas y de pequeña escala, destacándose la explotación de tropillas familiares y la existencia de eventos de caza en el lapso verano-otoño, aunque por el momento sin poder descartar la existencia de eventos ocurridos en otras estaciones. También en los casos para los que se dispone de información indican que las presas han sido faenadas y procesadas en forma completa e intensiva. Hasta el momento, Túnel I parece ser el único caso donde los procesos atricionales han tenido mayor incidencia, particularmente la acción de los carnívoros. Resta ampliar el espectro de las investigaciones a fin de establecer si tal situación fuera una particularidad de este caso o si se registran situaciones similares, ya sea en otros sitios u otros taxones. En síntesis: los resultados resaltan el carácter complejo de la interacción de agentes humanos y naturales en la conformación del conjunto final y suman nueva información al estudio de los modos tafonómicos y las estrategias de explotación del guanaco en contextos de la región del Beagle.

\section{AGRADECIMIENTOS}

Primero y muy especialmente quiero mencionar a Nadia Rua, con quien trabajamos largos meses revisando y comparando antiguas bases de datos y realizando una cuidadosa reclasificación de los materiales incluidos en este trabajo. Sin su colaboración este trabajo hubiese demandado muchísimo más tiempo y sin dudas hubiese cometido muchísimos más errores. Este artículo se ha visto sensiblemente mejorado por las sugerencias aportadas por: Luis Orquera, Ernesto Piana, Luis Borrero, Laura Miotti, Fernando Santiago, Jimena Oría y Francisco Zangrando, quienes leyeron distintas versiones del manuscrito. Por último debo mencionar a los dos evaluadores anónimos, quienes contribuyeron con constructivas observaciones. A todos ellos muchas gracias.

\section{BIBLIOGRAFÍA}

Alunni, D. (2013). Estrategias de transporte, procesamiento y consumo de guanacos en la costa sur de Tierra del Fuego (Argentina). Tesis de Licenciatura. Facultad de Filosofía y Letras. Universidad Nacional de Buenos Aires.

Alunni, D. \& Zangrando, A. F. (2012). Primeros datos sobre el transporte, procesamiento y consumo de guanacos en la localidad arqueológica Hershkaia (Sudeste de Tierra del Fuego, Argentina). Magallania, 40(1), 319-331.

Álvarez, M. R. (2004). Estrategias tecnológicas en los grupos canoeros tempranos del área fuego-patagónica. Magallania, 32, 191-208.

Behrensmeyer, A. K. (1978). Taphonomic and ecology information from bone weathering. Paleobiology, 4, 150-162.

Binford, L. (1978). Nunamiut Ethnoarchaeology. Academic 
Press, Nueva York.

(1981). Bones: ancient men and modern myths. Academic Press, New York.

Borrero, L. (1990). Fuego-Patagonia, Bone assemblage and problems of communal guanaco hunting. En Davis \& Reeves (Eds.), Hunters of the recent past. (pp. 373339). Unwin Hyman.

Borrero, L. (2007a). Longitudinal Taphonomic studios in Tierra del Fuego. En M. A. Gutierrez, L. Miotti, G. Barrientos, G. Mengoni Goñalons \& M. Salemme (Eds.), Taphonomy and Zooarchaeology in Argentina. (pp 219-233) BAR Internacional Series 1601. Archaeopress. Oxford.

Borrero, L. (2007b). No Direction Home: Vertebrate Taphonomy in Argentina. En M. A. Gutierrez, L. Miotti, G. Barrientos, G. Mengoni Goñalons \& M. Salemme (Eds.), Taphonomy and Zooarchaeology in Argentina. (pp 9-12). BAR International Series 1601. Archaeopress. Oxford.

Bunn, H. T. \& Kroll, E. M. (1986). Systematic butchery by Pilo/Pleistocene hominids at Olduvai Gorge, Tanzania. Current Anthropology, 27, 431-452.

Cleghorn, N. \& Marean, C. (2004). Distinguishing Selective Transport and In Situ Attrition: A Critical Reviewed of Analytical Approaches. Journal of Taphonomy, 2, 4367.

Elkin, D. (1995). Volume density of South American camelid skeletal parts. International Journal of Osteoarchaeology, 5, 29-37.

Emerson, A. M. (1993). The Role of Body Parts Utility in Small-scale Hunting under Two Strategies of Carcass Recovery. En J. Hudson (Ed.). From Bones to Behavior. Ethnoarchaeological and Experimental Contributions to the Interpretetation of Faunal Remains. (pp 138-155). Center for Archaeological Investigation.Southern Illinois University at Carbondale. Occasional Paper $\mathrm{N}^{\circ} 21$.

Estévez E., J. \& Vila, A. (1995). Encuentro en los conchales fueguinos. Treballsd' Etnoarqueologia $n^{\circ} 1$. CSIC $y$ Universidad Autónoma de Barcelona. Barcelona.

Estévez E., J. \& Mameli, L. (2000). Muerte en el Canal: experiencias bioestratinomicas controladas sobre la acción sustractora de Cánidos. Archaeofauna, Vol 9, 7-16.

Figuerero Torres, M. J. \& Mengoni Goñalons, G. (1986). Excavaciones arqueológicas en la isla El Salmón (Parque Nacional de Tierra del Fuego). Informes de Investigación 4. Buenos Aires. Programa de estudios Prehistóricos-CONICET.
Lyman, R. L. (1987). Archaeofaunas and butchery studies: a taphonomic perspective. In Advances in Archaeological method and theory. M. B. Schiffer (Ed.). (pp. 249-337). San Diego. Academia Press.

Lyman, R. L. (1992). Anatomical considerations of utility curves in zooarchaeology. Journal of Archaeological Science, 19, 7-22.

Lyman, R. L. (1994). Vertebrate Taphonomy. Cambridge Press.

Marean, C. (1995). Of Taphonomy and Zooarchaeology. Evolutionary Anthropoly, 4(2), 64-72.

Marean, C. y Cleghorn, N. (2003). Large Mammal Skeletal Element transport: Applying Foraging Theory in a Complex Taphonomic System. Journal of Taphonomy, 1(1), 15-42.

Marean, C. \& Frey, C. (1997). Animal bones from caves to cities. Reverse utillity curves as methodological artifacts. American Antiquity, 62, 698-716.

Marean, C. W. \& Spencer, L. M. (1991). Impact of carnivore ravaging on zooarchaeological measures of element abundance. American Antiquity, 56 (4), 645-658.

Mengoni Goñalons, G. L. (1986). Análisis de los vertebrados de Isla el Salmón. En Excavaciones arqueológicas en la isla El Salmón (Parque Nacional de Tierra del Fuego). Informes de Investigación 4 (pp 52-66). Buenos Aires. Programa de estudios PrehistóricosCONICET.

Mengoni Goñalons, G. L. (1999). Cazadores de guanacos de la estepa patagónica. Sociedad Argentina de Antropología, Buenos Aires.

Metcalfe, D. \& Jones, K. T. (1988). A reconsideration of Animal Body-Part Utility indices. American Antiquity, 53, 486-504.

Miotti, L. \& Marchionni, L. (2014). Autopodios de guanacos en sitios arqueológicos: equifinalidad entre lo palatable y lo preservado. Revista Chilena de Antropología, 29, 122-129

Muñoz, A. S. (2001). El guanaco en la dieta de los cazadoresrecolectores del norte de Tierra del Fuego. Análisis de dos casos procedentes del área Bahía Inútil-San Sebastián. En G. Mengoni Goñalons, D. Olivera \& H. Yacobaccio (Eds.). El uso de los camélidos a través del tiempo (pp 155-178). Ediciones del Tridente. Buenos Aires.

Muñoz, A. S. (2005). Zooarqueología del sector Atlántico de la Isla Grande de Tierra del Fuego. La utilización de camélidos y pinnípedos por los cazadores-recolectores fueguinos. Relaciones de la Sociedad Argentina de Antropología, XXX, 59-78. 
Muñoz, A. S. (2008). El procesamiento de los camélidos fueguinos en el pasado. Aspectos metodológicos y resultados alcanzados para el sector atlántico de Tierra del Fuego. En A. Acosta, D. Loponte, D. \& L. Mucciolo (Eds.), Estudios Tafonómicos y Zooarqueológicos (I) (pp. 77-97). Instituto Nacional de Antropología y Pensamiento Latinoamericano. Buenos Aires.

Muñoz, A. S. (2009). Los canidos como agentes tafonómicos en los depósitos arqueológicos de la Isla Grande de Tierra del Fuego. En M. Salemme, F. Santiago, M. Álvarez, E. L. Piana, M. Vázquez \& M. E. Mansur (Eds.) Arqueología de la Patagonia. Una mirada desde el último confín, Tomo II. (pp 799-811). Editorial Utopias. Ushuaia.

Orquera, L. A. (2005). Mid-Holocene littoral adaptation at the southern end of South America. Quaternary International, 132, 107-115.

Orquera, L. A. \& Piana, E. L. (1988). Composición tipología y datos tecnomorfologicos y tecnofuncionales de los distintos conjuntos arqueológicos del sitio Túnel I (Tierra del Fuego). Relaciones de la Sociedad Argentina de Antropología. XVII (I) (1986-1987), 201-239. Buenos Aires.

Orquera, L. A. \& Piana, E. L. (1992). Un paso hacia la resolución del palimpsesto. En L. A. Borrero \& J. L. Lanata (Comp.), Análisis espacial en la arqueología patagónica (pp. 21-52). Ayllu. Buenos Aires.

Orquera, L. A. \& Piana, E. L. (1996). El sitio Shamakush I (Tierra del Fuego, Republica Argentina). Relaciones de la Sociedad Argentina de Antropología, XXI, 215265.

Orquera, L. A. \& Piana, E. L. (1999). Arqueología de la región del Canal Beagle (Tierra del Fuego). Publicaciones de la Sociedad Argentina de Antropología. Buenos Aires.

Orquera, L. A. \& Piana, E. L. (2000). Composición de conchales de la costa del canal Beagle (Tierra del Fuego, República Argentina) Primera parte. Relaciones de la Sociedad Argentina de Antropología, XXV, 249-274.

Orquera, L. A. \& Piana, E. L. (2001). Composición de conchales de la costa del canal Beagle (Tierra del Fuego, República Argentina) Segunda parte. Relaciones de la Sociedad Argentina de Antropología, XXVI, 345-368.

Otaola, C. (2010). Índices de utilidad económica en contextos tafonómicos variados. En M. Gutierrez, M. De Nigris, P. Fernández, M. Giardina, A. Gil, A. Izeta, G. Neme \& H. Yacobaccio (Eds.), Zooarqueologia a principios del siglo XXI. Aportes teóricos, metodológicos y casos de estudio (pp 157-166). Ediciones Espinillo. Buenos Aires.

Piana, E. L. (1984). Arrinconamiento o adaptación en Tierra del Fuego. En Ensayos de Antropología Argentina (año 1984): 7-110. Ed. Univ. de Belgrano, Buenos Aires.

Piana, E. L. \& Canale, G. (1995). Túnel II: un yacimiento de la Fase reciente del Canal Beagle. Relaciones de la sociedad Argentina de Antropología, XIX (19931994), 363-389.

Piana, E. L. \& Vázquez, M. (2008). El sitio Shamakush VIII. Puntualizaciones sobre el uso de recursos y la gestión del asentamiento en el canal Beagle. En A. Austral \& M. Tamagnini (Comps.). Problemáticas de la Arqueología Contemporánea, Tomo III (pp 1057-1068). Editorial Universidad Nacional de Rio Cuarto. Fac. de Cs. Exactas, Físicas y Naturales. Rio Cuarto. Córdoba.

Piana, E. L., Vázquez, M. \& Tivoli, A. (2007). Dieta y algo más. Animales pequeños y variabilidad del comportamiento humano en el canal Beagle. En F. Morello, M. Martinic, A. Prieto \& G. Bahamondes (Eds.). Arqueología de Fuego-Patagonia, levantando piedras, desenterrando huesos... y develando arcanos (pp 39-50). Ediciones CECUA. Punta Arenas. Chile.

Piana, E. L., Vázquez, M. \& Álvarez, M. (2008). El sitio Ajej I: un aporte a la variabilidad de estrategias de los canoeros fueguinos. Runa, 29, 101-121.

Prevosti, F. J., Santiago F., Prates L. \& Salemme, M. (2011). Constraining the time of extinction of the South American fox Dusicyon avus (Carnivora, Canidae) during the late Holocene. Quaternary International, 245, 209-217.

Santiago, F. (2013). La ocupación humana del norte de Tierra del Fuego durante el Holoceno medio y tardio. Editora Cultural Tierra del Fuego, Ushuaia.

Santiago, F. \& Vázquez (2012). Dietas promediadas: explorando el registro zooarqueológico supra-regional en Tierra del Fuego. Revista del Museo de Antropología 5, 225238, Facultad de Filosofía y Humanidades - Universidad Nacional de Córdoba.

Schiavini, A. (1993). Los lobos marinos como recurso para cazadores-recolectores marinos: El caso de Tierra del Fuego. Latin American Antiquity, 4, 346-366.

Stiner, M. C. (1991). Human Predators and prey mortality. M. C. Stiner (Ed.). Boulder: Westview Press.

Tivoli, A. M. (2010). Exploitation of bird resources among prehistoric sea-nomad societies of the Beagle Channel region, southern South America. Before Farming 2010/2 article 3, 1-12.

Tivoli, A. M. (2012). ¿Intensificación? en el aprovechamiento de aves entre los cazadores-recolectores-pescadores de la región del canal Beagle. Archaeofauna, 21, 121-137. 
Tivoli, A. M. (2014). Las aves en la alimentación y tecnología de los pueblos originarios de la región del canal Beagle. En J. Oría, \& A. Tívoli (Eds.), Cazadores de mar y tierra. Estudios recientes en arqueología fueguina (pp 85107). Editora Cultural Tierra del Fuego y Museo del Fin del Mundo. Ushuaia.

Tivoli, A. M. \& Zangrando, A. F. (2011). Subsistence variations and landscape use among maritime hunter gatherers. A zooarcheological analysis from the Beagle Channel (Tierra del Fuego, Argentina). Journal of Archaeological Science, 38, 1148-1156.

Zangrando, A. F. J. (2003). Ictioarqueología del canal Beagle. Explotación de peces y su implicancia en la subsistencia humana. Sociedad Argentina de Antropologia. Buenos Aires.

Zangrando, A. F. J. (2009). Historia evolutiva y subsistencia de cazadores-recolectores marítimos de Tierra del Fuego. Colección Tesis Doctorales. Sociedad Argentina de Antropología, Buenos Aires.

Zangrando, A. F. J. (2014). Entre la caza y la pesca. Variaciones en el consumo de animales entre los pueblos originarios del canal Beagle en 6000 años de historia. En J. Oría \& A. Tivoli (Eds.), Cazadores de mar y tierra. Estudios recientes en arqueología fueguina (pp 67-84). Editora Cultural Tierra del Fuego y Museo del Fin del Mundo. Ushuaia. 
